\title{
The PXL1 Gene of Saccharomyces cerevisiae Encodes a Paxillin-like Protein Functioning in Polarized Cell Growth
}

\author{
Nancy A. Mackin, Tarek J. Sousou, and Scott E. Erdman* \\ Department of Biology, Syracuse University, Syracuse, New York 13244
}

Submitted January 5, 2004; Accepted January 22, 2004

Monitoring Editor: Trisha Davis

\begin{abstract}
The Saccharomyces cerevisiae open reading frame YKR090w encodes a predicted protein displaying similarity in organization to paxillin, a scaffolding protein that organizes signaling and actin cytoskeletal regulating activities in many higher eucaryotic cell types. We found that YKR090w functions in a manner analogous to paxillin as a mediator of polarized cell growth; thus, we have named this gene PXL1 (Paxillin-like protein $\underline{1}$ ). Analyses of pxl1D strains show that PXL1 is required for the selection and maintenance of polarized growth sites during vegetative growth and mating. Genetic analyses of strains lacking both PXL1 and the Rho GAP BEM2 demonstrate that such cells display pronounced growth defects in response to different conditions causing Rho1 pathway activation. PXL1 also displays genetic interactions with the Rho1 effector FKS1. Pxl1p may therefore function as a modulator of Rho-GTPase signaling. A GFP::Pxl1 fusion protein localizes to sites of polarized cell growth. Experiments mapping the localization determinants of Pxl1p demonstrate the existence of localization mechanisms conserved between paxillin and Pxl1p and indicate an evolutionarily ancient and conserved role for LIM domain proteins in acting to modulate cell signaling and cytoskeletal organization during polarized growth.
\end{abstract}

\section{INTRODUCTION}

In eucaryotes, polarized growth occurs through the concerted activity of several classes of polarity proteins that act to specify sites of cytoskeletal reorganization and membrane expansion. Two key classes of polarity proteins have been found to play ubiquitous roles in polarity establishment and maintenance. One key class of polarity regulators is the low-molecular-weight cytosolic GTPase proteins represented by the protein families of Ras, Rac, and Rho. The organized assembly and disassembly of these polarity regulating components is coordinated through a second class of polarity proteins whose members include a variety of scaffolding proteins. At present, the factors and mechanisms that organize and promote the efficient transduction of Rho dependent signals to appropriate subsets of pathway effector proteins remain in many cases to be determined, although at least some of these roles appear to be fulfilled by scaffolding proteins that aid in the correct organization of signaling proteins and their regulators.

Coordination of these polarity components is required for the formation of polarized growth structures in higher eucaryotes. Specifically, a number of LIM domain containing proteins such as paxillin, zyxin, and CRP act as molecular adaptors or scaffolds, linking integrin and growth factormediated signaling from the cell exterior to the actin cytoskeleton (Sadler et al., 1992; Turner, 2000). These proteins belong to a superfamily whose members possess LIM domains and represent a class of cytoskeletal, signaling, and transcription-regulating proteins that have been implicated

Article published online ahead of print. Mol. Biol. Cell 10.1091/ mbc.E04-01-0004. Article and publication date are available at www.molbiolcell.org/cgi/doi/10.1091/mbc.E04-01-0004.

*Corresponding author. E-mail address: seerdman@syr.edu. broadly in differentiation processes (Schmeichel and Beckerle, 1994; Bach, 2000; Khurana et al., 2002b). Furthermore, these proteins have been found to localize to focal contacts, sites of active signaling from the extracellular matrix to the internal cellular environment. At these highly dynamic sites of signaling, Rho proteins and their regulators enable formation of such polarized structures as stress fibers, filiopodia, and lamellipodia (Hall, 1998).

In Saccharomyces cerevisiae, a well-characterized cascade of events during polarized growth exists in which Ras-related and Rho family GTPases determine, establish, and maintain sites of growth (Chant, 1999). As in mammalian systems, cell surface markers participate in the transduction of signals, resulting in actin cytoskeletal reorganization and changes in gene expression. During budding, filamentous growth and mating, cells utilize cortical cues (landmarks) that signal local recruitment of a set of polarity establishment proteins, including scaffold proteins, which create plasma membraneassociated domains that promote formation and maintenance of F-actin structures. The actin cytoskeleton and associated myosin V-dependent vesicle targeting functions direct secretory vesicle deposition to these cortical domains (Pruyne and Bretscher, 2000; Bretscher, 2003). During budding, these domains form first where a bud will emerge (incipient bud site) and subsequently remain tightly associated with the tip of the small growing bud.

Growth landmarks in yeast represent cortical domains that are specified and recognized by the activities of septin proteins and BUD genes, which encompass a variety of cell type-dependent and bud site selection-independent functions. The BUD 1, 2, and 5 loci comprise a Ras-related GTPase module specifying growth sites in all vegetative cells, and in some situations mating cells (Dorer et al., 1997; Chant, 1999). BUD3 and 4, AXL1, and AXL2/BUD10 control bud placement in haploid cells, and an extensive group of 
other BUD genes, along with many actin-related functions, act to specify bud growth sites in diploid cells (Zahner et al., 1996; Ni and Snyder, 2001; Casamayor and Snyder, 2002). As buds mature, polarized growth next occurs isotropically in the bud and then finally at the mother-bud neck where cytokinesis occurs. During mating, growth sites are continuously specified by local areas of pheromone receptor activation that recruit polarity components to target secretion to the tip of mating cells, a structure termed the mating projection (or shmoo; Herskowitz et al., 1995; Pringle et al., 1995; Arkowitz, 1999). In each of these cases, growth is catalyzed by the action of Rho-GTPases that are recruited to these sites where they control different effectors that act to modulate cortical actin, cell wall synthesis enzymes, and other targets controlling cell polarity (Chant and Stowers, 1995; Cabib et al., 1998; Schmidt and Hall, 1998). The activities of growth targeting landmark proteins are also thought to stimulate the local assembly or scaffolding of polarity proteins such as Bni1p, Aip3/Bud6p, and Spa2p that in turn may help maintain the localization of the Rho GTPases and their effectors (Fujiwara et al., 1998; Nern and Arkowitz, 1998, 1999; Arkowitz, 1999; van Drogen and Peter, 2002). Similarly, changes in cell integrity can also serve to activate Rho signaling through stress-sensitive sensors (Nobes and Hall, 1995; Bickle et al., 1998; Delley and Hall, 1999; Galbraith et al., 2002).

The Rho GTPase proteins themselves function as molecular switches, relaying signaling information to target effector proteins that include other signaling molecules, regulators of actin assembly and vesicle docking (reviewed in Nobes and Hall, 1995; Tapon and Hall, 1997; Johnson, 1999; Etienne-Manneville and Hall, 2002). In budding yeast, two of the six Rho-type GTPases encoded by the genome, CDC42 and RHO1, are each essential for polarized growth and therefore viability (Adams et al., 1990; Drgonova et al., 1996; Johnson, 1999). The activities of these proteins are determined by their nucleotide bound state and are regulated by GEFs (GDP to GTP exchange promoters), GAPs (GTPase activators), and GDIs (guanine nucleotide dissociation inhibitors; reviewed in Johnson, 1999). Of the six yeast Rhorelated GTPases, the regulators and effectors of CDC42 and RHO1 have been the most thoroughly investigated; relatively modest phenotypes of other RHO genes and their effectors have left more of their functions and regulation remaining to be understood. Genetic studies of CDC42 and RHO1 functions have led to a picture in which polarized growth is initiated in restricted domains by Cdc $42 p$ and maintained subsequently and principally through the activation of Rho1p. At least some of the regulators of Rho GTPases, such as the GEFs Cdc24p and Rom2p and the GAP Lrg1p, are also known to localize to polarized growth sites (Manning et al., 1997; Toenjes et al., 1999; Watanabe et al., 2001).

Here we describe a novel modulator of yeast cell polarity that possesses similarities in primary sequence, structural organization, and function to the mammalian scaffolding protein paxillin. We have named the open reading frame encoding this protein, YKR090w, Paxillin-like protein $\underline{1}$ or PXL1. Genetic analyses involving pxl1s cells indicate that PXL1 functions in polarized cell growth in both vegetative and mating yeast cells, most likely by modulating Rho GTPase-mediated signaling. Consistent with such a function, Pxl1p localizes to sites of polarized growth in yeast. Localization of Pxl1p to these growth sites requires the activities of conserved LIM domains found within the carboxy-terminus of Pxl1p, in a manner that depends on unique targeting roles of each LIM domain. The roles performed by the Pxl1p LIM domains in growth site targeting are generally similar to the functions played by homologous LIM domains found in paxillin and a number of other related group 3 LIM proteins present in other eukaryotes.

\section{MATERIALS AND METHODS}

\section{Yeast Strains, Growth, and Genetic Manipulation}

Yeast strains used in this study are listed in Table 1. Growth media and genetic manipulations were as described by Sherman et al. (1986). Yeast transformations were performed using the one-step method (Chen et al., 1992). A complete deletion of PXL1 in YSE20 was constructed using a PCR disruption procedure (Baudin et al., 1993). Oligonucleotides containing the 55 base pairs immediately upstream of the initiation codon and downstream of the termination codon of PXL1 were synthesized with the corresponding end sequences: 5'-AGGCGCGTTTCGGTGATGACGGTG and 5'-AGGGTGATGGTTCACGTAGTGGGC, respectively. These sequences are complementary to regions that flank the URA3 gene of pRS306. Fragments containing URA3 flanked by PXL1 sequences were amplified by PCR and transformed into the diploid strain YSE20. Strains containing URA3 replacements of PXL1 were confirmed by PCR analysis. Two independently derived replacement alleles were confirmed, these heterozygotes were sporulated, and haploid segregants containing the PXL1 disruption were analyzed in phenotypic assays.

\section{Analyses of Bud Site Selection and Mating Cell Polarization}

For bud-site selection analyses, cultures were grown overnight in YPAD medium. Cells, $1.5 \mathrm{ml}$, were pelleted by centrifugation and washed once with $500 \mu \mathrm{l}$ of distilled water and once with $1 \times$ PBS. Cells were resuspended in 400 $\mu \mathrm{l}$ of $1 \times$ PBS. To the suspension, $200 \mu \mathrm{l}$ of $1 \mathrm{mg} / \mathrm{ml}$ Calcofluor white (Sigma, St. Louis, MO) was added. Cells were stained for $10 \mathrm{~min}$, washed twice in $1 \times$ PBS, and resuspended in $400 \mu \mathrm{l}$ of $1 \times$ PBS. Twenty-five microliters of each cell suspension was then pelleted and resuspended in $5 \mu \mathrm{l}$ of mounting solution (70\% glycerol, 30\% PBS, $2 \% \mathrm{wt} / \mathrm{vol} n$-propyl gallate). Cells were visualized using an inverted Nikon Eclipse TE300 microscope (Garden City, NJ) set up for differential interference contrast (DIC) and epifluorescence viewing. Calcofluor staining was viewed using a standard UV-DAPI filter set. Haploid and diploid cells possessing two or three bud scars were classified into two categories according to bud scar position: polar (scars at one or both poles) or mixed (at least one of the two or three scars found in the medial region of the cell). Analyses of two independently constructed $p x l 1 \Delta$ strains yielded results highly similar to those presented for the diploid strain constructed from the one transformant described in the text

To assay mating cell polarization, cultures grown overnight in YPAD medium were diluted to an optical density at $600 \mathrm{~nm}\left(\mathrm{OD}_{600}\right)$ of 0.2 in fresh YPAD medium and grown to an optical density at $600 \mathrm{~nm}\left(\mathrm{OD}_{600}\right)$ of 0.4 . Cells were mixed and allowed to mate in liquid medium at 30 or $37^{\circ} \mathrm{C}$ for $4 \mathrm{~h}$, formaldehyde-fixed, and processed for microscopy as described in Erdman et al. (1998). Mating cell morphologies were viewed using a Nikon Eclipse TE300 microscope under DIC illumination. Mating projections morphologies were scored as follows: wild type, blunt, and hyperpolarized as defined in Erdman et al. (1998). Cells from mating mixtures were also stained with FM4-64 and mounted for viewing in glycerol/PBS containing $0.0225 \mu \mathrm{g} / \mathrm{ml}$ DAPI $\left(4^{\prime}, 6-\right.$ diamidoino-Z-phenylindole) to allow quantification of cell-cell fusion in crosses involving pxl1s cells in comparison to wild-type strains. As with the bud site selection analyses, examination of two independently constructed pxl1s strains yielded results very similar to those presented for the one transformant strain described in the text.

\section{Constructions of Plasmids Containing PXL1 Sequences}

Plasmids used in this study are listed in Table 2. Standard cloning procedures were followed according to Sambrook et al. (1989). A plasmid, pBSE352, carrying the green fluorescent protein (GFP) coding sequences fused to the PXL1 coding sequences, was constructed by first cloning a PCR fragment containing 1000 base pairs of the promoter region of the PXL1 locus flanked by the restriction sites XhoI and NotI into the yeast centromeric shuttle vector pRS315. Subsequently, a 2.6-kb PCR fragment consisting of the PXL1 coding sequences preceded by NotI and SalI sites introduced in the PCR primer and an additional 500 base pairs downstream of the coding sequence followed by an introduced SacI site, was inserted into the former construct restriction digested with NotI and SacI. Finally, a 714-base pair PCR fragment containing the GFP coding sequences flanked by NotI and SalI sites was introduced into this construct digested with these restriction enzymes. All PCR reactions were performed using Vent high-fidelity polymerase (New England Biolabs, Beverly, MA). Genomic DNA templates were prepared from S288c-derived strains. Normal function of this GFP:Pxl1p fusion was confirmed by the ability of the plasmid to complement the pxl1 $\Delta$ dependent conditional growth defects observed in a bem $2 \Delta$ pxl1s strain (YSE838) as described in the text. This plasmid fully rescued these defects on synthetic complete media containing SDS (sodium dodecylsulfate). 
Table 1. Strains used in this study

\begin{tabular}{|c|c|c|}
\hline Strain ${ }^{a}$ & Genotype & Source \\
\hline YSE 20 & 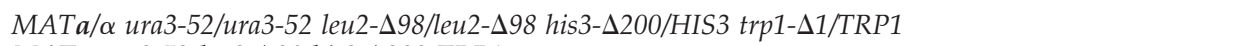 & Y800; Burns et al. (1994) \\
\hline YSE 21 & 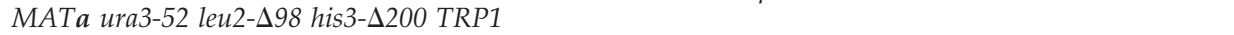 & \\
\hline YSE 536 & 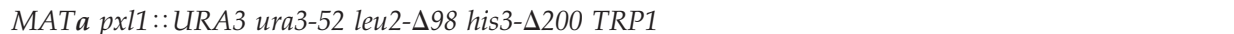 & \\
\hline YSE 537 & MAT $\alpha$ pxl1::URA3 ura3-52 leu2- $\Delta 98$ HIS3 trp1- $\Delta 1$ & \\
\hline YSE 538 & 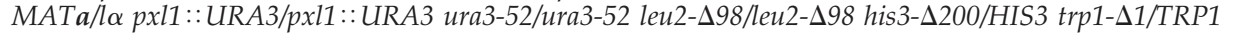 & \\
\hline YSE 430 & MATa ura $3 \Delta 0$ leu $2 \Delta 0$ met $15 \Delta 0$ LYS2 his $3 \Delta 1$ TRP1 & Winzeler et al. (1999) \\
\hline YSE 431 & MAT $\alpha$ ura3 $\Delta 0 l e u 2 \Delta 0$ MET15 lys $2 \Delta 0$ his $3 \Delta 1$ TRP1 & \\
\hline YSE 554 & 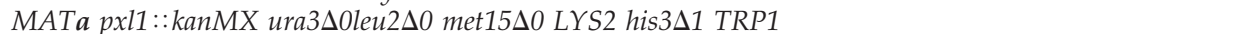 & \\
\hline YSE 555 & 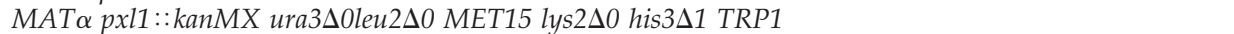 & \\
\hline YSE 763 & MATa $c d c 12-6$ ts & Hartwell (1971) \\
\hline YSE 865 & MATa ura3-52 leu2 21 his3- $\Delta 200$ sec14-3 & Jin and Amberg (2000) \\
\hline YSE 866 & MATa ura3-52 leu2 $\Delta 1$ his3- $\Delta 200$ sec17-1 & \\
\hline YSE 867 & 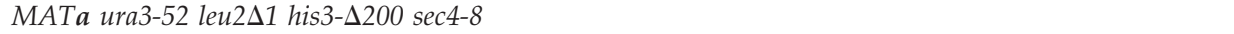 & \\
\hline YSE 800 & 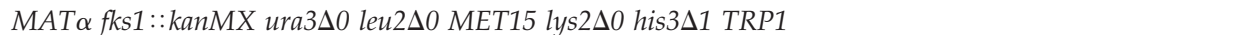 & \\
\hline YSE 801 & 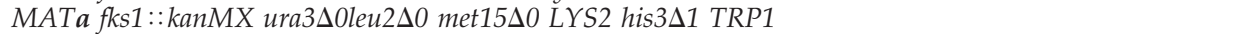 & \\
\hline YSE 804 & 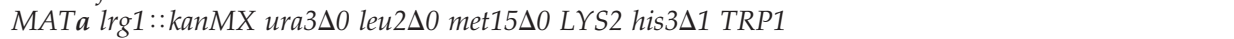 & \\
\hline YSE 805 & 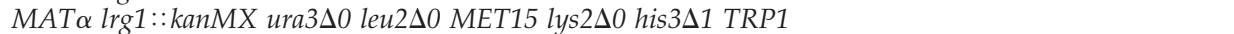 & \\
\hline YSE 814 & 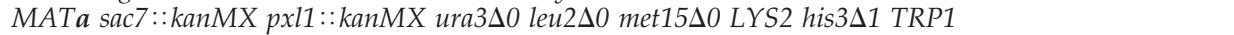 & \\
\hline YSE 816 & 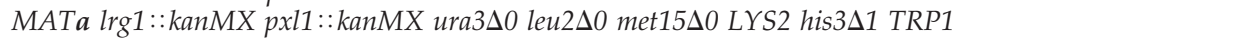 & \\
\hline YSE 818 & 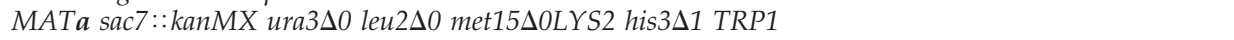 & \\
\hline YSE 819 & 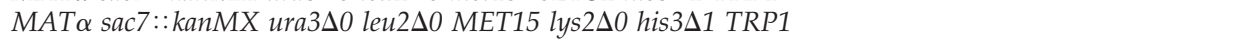 & \\
\hline YSE 828 & 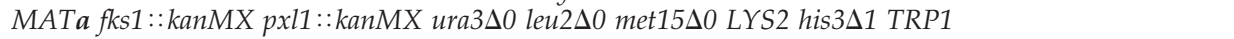 & \\
\hline YSE 838 & 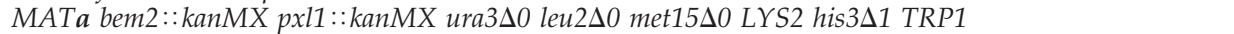 & \\
\hline YSE 661 & MATa bem $2:: k a n M X$ ura3s0 leu $2 \Delta 0$ met $15 \Delta 0$ LYS2 his $3 \Delta 1$ TRP1 & \\
\hline YSE 663 & MAT $\alpha$ bem $2:: k a n M X$ ura $3 \Delta 0$ leu $2 \Delta 0$ MET15 lys $2 \Delta 0$ his $3 \Delta 1$ TRP1 & \\
\hline YSE 637 & MATa rom $2:: k a n M X$ ura $3 \Delta 0$ leu $2 \Delta 0$ met $15 \Delta 0$ LYS2 his $3 \Delta 1$ TRP1 & \\
\hline YSE 639 & $M A T \alpha$ rom $2:$ kanMX ura3 $\Delta 0 l e u 2 \Delta 0$ MET15 lys $2 \Delta 0$ his $3 \Delta 1$ TRP1 & \\
\hline YSE 665 & 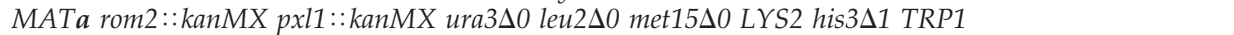 & \\
\hline
\end{tabular}

a All strains were constructed for this study unless otherwise indicated; sources of kanMX alleles are strains described in Winzeler et al. (1999).

To construct a GFP fusion to the N-terminal 502 residues of Pxl1p, pBSE365, a stop codon was inserted following amino acid residue 502. Primers: 5'ATACTGCAGTAGGATCCCATAAGCGCAACATAACAGATCTG and 5-ATAGAGCTCTTAAGACTCTCGATTTGGGAGTC were used to amplify a 624base pair fragment, containing a PstI site followed by the stop codon TAG and the restriction sites BamHI and $\mathrm{SacI}$, respectively. This fragment was introduced into pBSE352 using the unique PstI site present within the PXL1 coding region and the SacI restriction site used to construct the pBSE352 GFP-PXL1 clone. To confirm the insertion of the stop codon following the PstI site within the PXL1 gene, a restriction digest was performed to establish the presence of an introduced, diagnostic BamHI site immediately following the PstI site, confirming insertion of the termination codon containing fragment.

A GFP fusion to the final 206 amino acid residues of Pxl1p, pBSE364, was made by replacement of the PXL1 coding sequence in the pBSE352 GFP-PXL1 construct with a 1118-base pair fragment containing the final 618 base pairs of the PXL1 coding sequence and an additional downstream 500 base pairs. Using the primer pair: 5'-ATAGTCGACCAACTGCAGCATAAGCGCA and 5'-ATAGAGCTCTTAAGACTCTCGATTTGGGAGTC. The fragment was amplified and inserted into pBSE352 via the restriction sites SalI and SacI. Mutagenesis of LIM domains 1 and 2 of Pxl1p was performed using fusion

Table 2. Plasmids used in this study

\begin{tabular}{|c|c|c|}
\hline Plasmid & Contents & Source \\
\hline BSE 19 & pRS315 & Sikorski and Hieter (1989) \\
\hline BSE 119 & pRS425 & Christianson et al. (1992) \\
\hline BSE 345 & PXL1 gene in pRS315: $-1000 /+500$, LEU-based plasmid, cloned Xho1-Sac1 & \\
\hline BSE 346 & $\begin{array}{l}\text { PXL1 gene in } 2 \text { micron vector pRS425; }-1000 /+500 \text {, LEU-based plasmid, cloned Xho1- } \\
\text { Sac1 }\end{array}$ & \\
\hline BSE 352 & $\begin{array}{l}\text { pRS315 containing GFP ::PXL1 and }-1000 \text { bp of upstream and }+500 \mathrm{bp} \text { of downstream } \\
\text { PXL1 }\end{array}$ & \\
\hline BSE 364 & $\begin{array}{l}\text { GFP-pxl1-LIM only clone, constructed by removing PXL1 coding sequences for a.a. 1-502 } \\
\text { from BSE352 }\end{array}$ & \\
\hline BSE 365 & $\begin{array}{l}\text { GFP-pxl1-N terminus clone, constructed by inserting a nonsense codon into PXL1 in } \\
\text { BSE352 }\end{array}$ & \\
\hline BSE 378 & $\begin{array}{l}\text { pxl1-lim1; PXL1-H5811/C584A site-directed mutagenesis clone of LIM domain } 1 \\
\text { substituted in BSE345 }\end{array}$ & \\
\hline BSE 379 & $\begin{array}{l}\text { pxl1-lim2; PXL1-H6421/C645A site-directed mutagenesis clone of LIM domain } 2 \\
\text { substituted in BSE345 }\end{array}$ & \\
\hline BSE 385 & GFP-Pxl1-H5811/C584A site-directed mutagenesis clone substituted in BSE352 & \\
\hline BSE 386 & GFP-Pxl1-H6421/C645A site-directed mutagenesis clone substituted in BSE352 & \\
\hline
\end{tabular}

All plasmids were constructed for this study unless otherwise indicated. 


\section{Pxl1p}

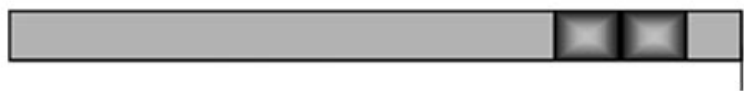

Paxillin

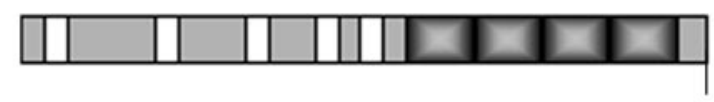

Figure 1. (A) Schematics representing the structural organization of Pxl1p and paxillin. Shaded and solid boxes indicate LIM domains and LD repeats, respectively. Predicted protein lengths are shown. (B) Alignment of Pxl1p LIM domains and the LIM domains three and four of paxillin alpha. Numbers indicate amino acid residues. Boldface cysteine and histidine residues indicate the LIM domain consensus sequence. Symbols show amino acid identities (:) and similarities $(+)$ between Pxl1p and paxillin family members.
B

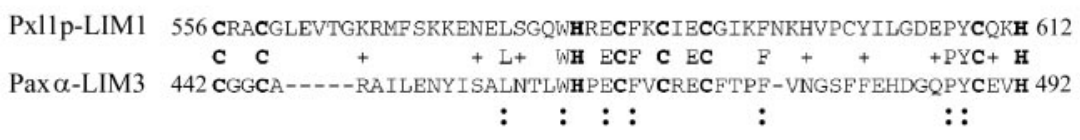

PX11p-LIM2 613 YHEENHSICKVCSNF IEGECLENDKVERFHVDCLNCFLCKTAITNDYYIFNGEIPLCGNH 672 $\mathrm{YHE} \mathrm{S}+\mathrm{C}$ C I G C $++\mathrm{FH}+\mathrm{C} \mathrm{C}+++\mathrm{Cl} \mathrm{N}^{+}$

Pax $\alpha$-LIM4 493 YHERRGSLSGCOKPITGRCI-TAMAKKFHPAHFVCAFCLKOLNKGTFKEONDKPYCONC 551
PCR. Mutagenic primers: 5'-CTGTCGGGTCAATGGATCCGTGAAGCTTTTAAATGCATTGAATGTGGTATC and 5'ATTCAATGCATTTAAAAGCTTCACGGATCCATTGACCCGACAGTTC were used to change histidine residue 581 to isoleucine and cysteine residue 584 to alanine within LIM domain 1. A fusion PCR fragment joining the mutagenized ends of the parent PCR fragments was amplified using a denatured mixture of the parent PCR fragments created with the mutagenic primers and the external primers 5'-ATACATATGGTCGACATGCTGACTCAAAATGACAC and 5'-ATAGAGCTCTTAAGACTCTCGATTTGGGAGTC. The resulting product was cloned into pBSE365 after digestion with the restriction enzymes Pst I and SacI. Similarly, mutagenesis of LIM domain 2 was performed using the mutagenic primers: 5'-AAGGTGGAACGATTCATTGTGGATGCTCTAAAACTGTT-TCTTATGCAAGACTGC and 5'-AGTCTTGCATAAGAAACAGTTTAGAGCATCCACAATGAATCGTTCCACCTTATC. The primers encode altered codons that change histidine residue 642 to isoleucine and cysteine residue 645 to alanine within LIM domain 2. Amplification of the fusion fragment and cloning was as done for mutagenesis of LIM1. LIM domain mutagenesis was confirmed by restriction digest with BamHI. Absence of the previously introduced BamHI site in pBSE365 confirmed the mutagenesis of LIM1 (pBSE341) or LIM2 (pBSE342). The manipulated regions of these constructs were subsequently DNA sequenced to confirm the presence of the intended mutations.

\section{Fluorescence Microscopy}

Fluorescence microscopy of yeast cells was performed as follows. Cultures of strains containing plasmid-based GFP fusions to PXL1 were grown overnight at $25^{\circ} \mathrm{C}$ in synthetic complete (SC) medium lacking the amino acid leucine (SC-leu) and supplemented with 1/10 volume $10 \mathrm{mM}$ adenine. Aliquots of cultures were placed on glass slides under coverslips, and GFP fluorescence was visualized using a Nikon Eclipse TE300 microscope with DIC and epifluorescence attachments and standard filter sets for UV-DAPI, Texas Red, and FITC using a $100 \times 1.3$ NA PlanApochromat objective. Excitation of GFP and image capture was done through the FITC filter set using a $5 \mathrm{MHz}$ CCD camera (Roper-Princeton Instruments, Princeton, NJ) and contrast in images adjusted using Adobe Photoshop (San Jose, CA) software. For experiments involving temperature shift of sec pathway mutants or drug treatment (latrunculin B; A.G .Scientific Inc., San Diego, CA), cells were treated for the indicated times before imaging an aliquot of the cells at the indicated times. Latrunculin B final concentration was $100 \mu \mathrm{M}$ (Marquitz et al., 2002); an equivalent amount of DMSO was added to untreated, control cultures. Imaging of YFP-Kex2p and a CFP-pxl-N terminal domain fusion was done with an Olympus BX microscope (Success, NY) outfitted with filter cubes for detection of these signals without channel cross-talk.

\section{Phenotypic Assays}

To examine genetic interactions between bem $2 \Delta$ and $f k s 1 \Delta$ mutations and pxl1s, strains were cultured, diluted to an optical density at $600 \mathrm{~nm}\left(\mathrm{OD}_{600}\right)$ of 1.0 , then serial dilutions by factors of 10 were made in $1.5-\mathrm{ml}$ sterile tubes, and the diluted cultures were transferred to a $96 \times 2-\mathrm{ml}$, "deep" well growth box. The box was placed on an inclined roller drum at room temperature for $10 \mathrm{~min}$ to ensure complete suspension of all strains. The diluted strains were then replica pinned onto YPAD plates or SC-leu plates (depending on the whether a plasmid was present in the strain) containing $5 \mu \mathrm{g} / \mathrm{ml}$ calcofluor, $10 \mu \mathrm{g} / \mathrm{ml}$ calcofluor, $0.005 \%$ SDS, or $0.01 \%$ SDS that were preincubated at 25 30 , or $37^{\circ} \mathrm{C}$. After inoculation, plates were placed at their respective temperatures for 3 to 4 days after which plate images were captured.

The fraction of inviable cells present in bem $2 \Delta$ pxl1s cultures at different temperatures relative to other strains was determined by staining of cells with the dye methylene blue as described in Iida et al. (1994) and Zhang et al. (2002), with the exception that cell aliquots were stained for $15 \mathrm{~min}$ in an equivalent amount of $0.01 \%$ methylene blue before visualization of stained and unstained cells by DIC microscopy. For the experiment assessing the viability of bem $2 \Delta$ pxl1s cells containing plasmids corresponding to PXL1, an empty vector or versions of PXL1 mutated in either of the two LIM domains, strains were grown overnight at $37^{\circ} \mathrm{C}$ to midlogarithmic phase, and the fraction of viable cells was assessed as described above.

\section{RESULTS}

\section{A Protein Similar to the Molecular Adaptor Protein Paxillin Is Encoded by the Yeast Genome}

We noted the presence of a predicted open reading frame in the yeast genome, YKR090w, which is characterized by specific sequence identities and a generally similar organization to the paxillin family of molecular adaptor signaling and cell polarity proteins. Further analyses of the predicted a 706amino acid long protein encoded by the open reading frame YKR090w using the sequence comparison programs BLAST and Clustal (Higgins and Sharp, 1988; Altschul et al., 1990), confirmed the presence of cysteine-rich sequence motifs matching the LIM domain consensus (Figure 1A). LIM domains are highly conserved double zinc-finger 

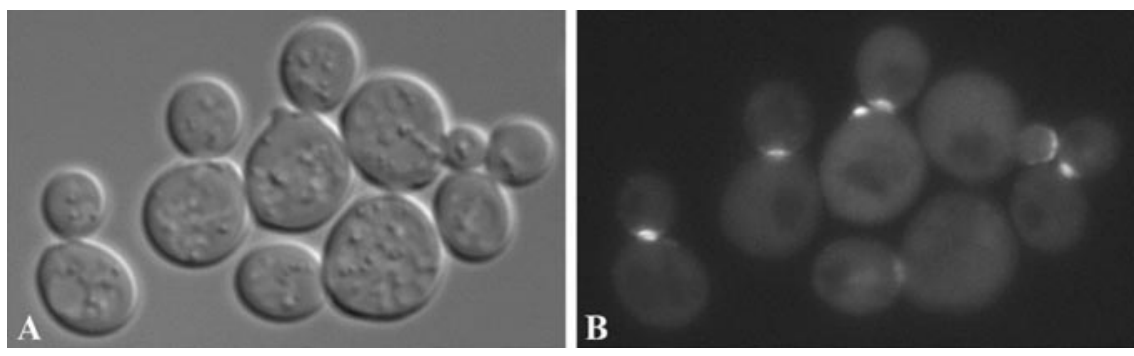

Figure 2. Pxl1p localizes to sites of polarized growth. A MATa $p x l 1 \Delta$ strain (YSE554) expressing a GFP::Pxl1p fusion was examined using differential interference contrast microscopy (A) and epifluorescence microscopy (B). The localization of GFP::Pxl1p to polarized growth site locations can be seen to be cell cycle stage dependent.

motifs that are comprised of the consensus sequence $\mathrm{CX}_{2} \mathrm{CX}_{16-23} \mathrm{HX}_{2} \mathrm{CX}_{2} \mathrm{CX}_{2} \mathrm{CX}_{16-21} \mathrm{CX}_{2-3}(\mathrm{C}, \mathrm{H}$, or D), where $\mathrm{X}$ is any amino acid. The predicted YKR090w gene product contains two carboxy-terminal LIM domains located between residues 556 and 612 and 621 and 672 . Among the different LIM protein superfamily members, the presence of C-terminal LIM domains classifies this protein as a Group 3 LIM protein (Taira et al., 1995; Jurata and Gill, 1998). Group 3 LIM protein members include paxillin and zyxin, two proteins that function as scaffolds for the regulated assembly of multiprotein complexes of signaling regulators and targets of the activities of Rho GTPase effectors at focal contact sites. Closer inspection of similarities between the two LIM domains of YKR090w and those of paxillin indicates that they share $31 \%$ identity and $44 \%$ similarity to LIM domains three and four present within the human paxillin homologue and its tissue-specific isoforms: paxillin beta and paxillin gamma (Figure 1B). Thus, YKR090w can be placed within this subgroup of LIM family genes based on structural and sequence homology. Based on its similarities to paxillin in structural organization, LIM domain sequences, and functions in polarized cell growth that we describe in this work, we have named the YKR090w gene Paxillin-like protein $\underline{1}$, or PXL1.

Beyond their similar LIM domain regions and carboxy terminal location characteristic of group 3 LIM family members, few similarities exist between the PXL1 gene product, Pxllp, and paxillin. Significant sequence similarities were not found by BLAST comparisons between the pre-LIM domain of Pxl1p and other eucaryotic protein sequences present in current databases, including those of paxillin and its family members. Comparison of cognate sequences of Pxl1p in other Saccharomyces species reveals a limited number of short domains within the pre-LIM amino terminal domain of Pxl1p that are conserved in these species (Cliften et al., 2003). Collectively, these findings are consistent with previous observations that this general class of LIM protein evolves rapidly in pre-LIM domain regions (Goyal et al., 1999).

\section{Pxl1p Localizes to Sites of Polarized Growth}

The roles of paxillin during polarized growth at focal contact sites led us to further explore the potential involvement of Pxl1p in polarized growth functions in budding yeast. Initially, we examined whether the protein localizes to sites of polarized growth. A fusion construct was generated containing the coding sequences for GFP (green fluorescent protein) fused to the full-length PXL1 coding sequences, expression of this construct remains under the control of the native PXL1 promoter (MATERIALS AND METHODS). Plasmids carrying this fusion gene were introduced into both wildtype and $p x l 1 \Delta$ strains and localization patterns examined in both haploid and diploid cells during vegetative growth. In unbudded cells, GFP::Pxllp can be seen to localize as a dot near the periphery of the cell at polar locations predicted to be incipient bud sites. In small-budded cells, GFP::Pxl1p appears as a crescent at the tip of the bud, and in largebudded cells, it is found at the mother-bud neck (Figure 2). We also observed the localization of GFP::Pxllp in cells forming mating projections. In pheromone-treated cells the GFP::Pxl1p fusion localizes to the tip of the mating projection (our unpublished results). These localization patterns during vegetative growth and mating are reminiscent of other cytosolic polarity components such as the RhoGTPases Rho1p and Cdc42p, as well as other polarity establishment proteins including Spa2p, Bem1p, Bni1p, Aip3/ Bud6p, Rom2p, and Lrg1p. Thus, Pxl1p localizes to sites of polarized growth in a pattern that is cell cycle dependent, suggesting its involvement in regulating or organizing other polarity proteins that function at these sites.

In paxillin, LIM domains 2 and 3 function to target the protein to focal adhesions (Brown et al., 1996). We noted that LIM1 of Pxl1p shares greatest homology with LIM3 of paxillin, the LIM domain contributing most significantly to paxillin focal contact targeting. On the basis of these similarities, we investigated the role(s) of the LIM domains of Pxl1p in targeting of the protein to polarized growth sites. To determine whether the LIM domains are necessary for targeting of Pxl1p, we introduced a nonsense codon into the full length GFP::Pxl1p fusion construct at a site corresponding to amino acid 503 of Pxl1p. The resulting construct of GFP fused to the N-terminal 502 amino acids of Pxl1p and lacking the two LIM domains, (GFP::pxl1-1-502) no longer localized to sites of polarized growth, irrespective of whether the cells contained or lacked endogenous wild-type Pxl1 protein. Instead, we observed a small patch-like intracellular localization pattern for the protein (Figure 3, A-C). Although this localization is somewhat reminiscent of that observed for Golgi compartment resident proteins in yeast, a colocalization experiment performed with YFP and CFP fusions Kex2p and px11-1-502, respectively, indicated that these proteins are not colocalized (our unpublished results). Alternatively, it is possible that the patches represent aberrant aggregates of this portion of the protein. Thus, whether the N-terminal region of Pxl1p associates with a defined intracellular compartment other than regions of the Golgi in which Kex $2 p$ is resident, and what the identity of that compartment may be, remains to be determined.

To test the ability of the LIM domain region of Pxl1p alone to mediate targeting of the protein to polarized growth sites, we constructed a fusion of GFP to final 206 amino acids of Pxl1p (GFP::pxl1-206-706), a region containing LIM1 and LIM2 of Pxl1p. As shown in Figure 4, A-C, we observed this protein to localize in unbudded, small-budded, and medium to large-budded cells with the cell cycle-dependent localization patterns characteristic of the full-length GFP::Pxl1p fusion. As shown, this occurs in pxl1s cells, indicating that localization of the minimal LIM domain-containing protein 
Figure 3. The preLIM domain of Pxl1p localizes to punctate intracellular domains. (A and B) Wild-type and (C) pxl1 $\Delta$ cells carrying the plasmid GFP::pxl1-1-502, encompassing the pre-LIM domain of Pxl1p but lacking the C-terminal LIM domains, were examined by epifluorescence microscopy for localization of the fusion protein. The fusion protein is associated with several punctate patches that lie within the interior of the cells.
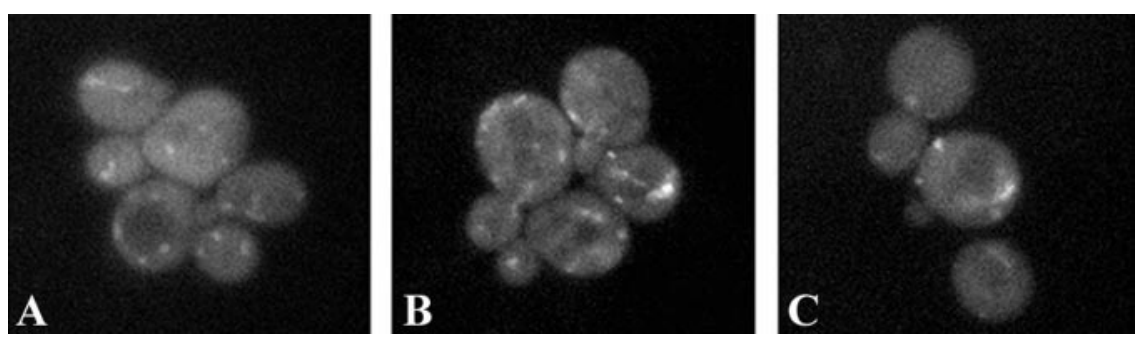

did not occur by association of the fusion protein with the endogenous wild-type protein. Collectively, these experiments demonstrate that the LIM domains of Pxl1p are both necessary and sufficient to target the protein to sites of polarized growth.

\section{PXL1 Participates in Selection of Polarized Growth Sites and Mating Morphogenesis}

To determine if, similar to paxillin, PXL1 is involved in directing or regulating polarized growth, we examined both haploid and diploid cells in which the PXL1 gene was completely replaced by either the nutritional marker URA3 or a heterologous drug resistance marker encoded by a kanMX cassette. Comparison of the growth rates of pxl1s cells to wild-type cells showed no differences in growth rate or cell morphologies at all temperatures tested (MATERIALS AND

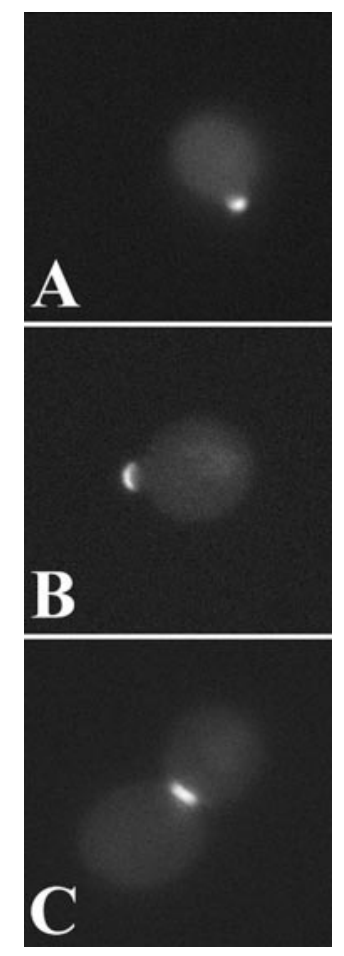

Figure 4. The LIM domain containing C-terminus of Pxl1p is sufficient to target the protein to sites of polarized growth. (A-C) pxl1 1 cells carrying the plasmid GFP::pxl1-503-706 that lacks the preLIM domain of Pxl1p were examined at different stages of the cell cycle for the distribution of the fusion protein. GFP::pxl1-503-706 localizes to the incipient bud site (A), the growing bud tip (B), and the mother-bud neck (C), in a manner identical to the localization pattern of the full-length GFP::Pxl1p fusion protein.
METHODS). Because a variety of genes encoding key polarity proteins (e.g., SPA2 and AIP3/BUD6) do not confer measurable growth rate defects on strains, but significantly affect the ability of cells lacking them to correctly localize cell growth in an efficient and properly spatially controlled manner, we tested whether pxl1 $\Delta$ strains were defective in other aspects of polarized cell growth.

Bud formation occurs at programmed sites in haploid and diploid cells. Based on the mating type of the cell, bud formation either follows an axial budding pattern or a bipolar budding pattern. Haploid cells bud in an axial pattern, placing buds exclusively at one pole of the cell, adjacent to previous sites of cell division, whereas, diploid cells follow a bipolar budding pattern in which budding may be initiated from either pole of the cell. Previous studies have shown that, specifically in diploid cells, mutations in actinregulating proteins and actin itself are associated with defects in bipolar bud site selection fidelity. To examine bud site selection patterns, cultures of haploid and homozygous diploid wild-type and pxl1s cells were grown to midlogarithmic phase, fixed, and stained with the chitin-binding dye, calcofluor white, which decorates bud scars or previous sites of bud formation and cytokinesis. Haploid pxl1s cells possessed an axial budding pattern essentially identical to wild-type cells. However, in diploid homozygous pxl1s cells, an aberrant budding pattern was frequently observed. In contrast to the high-fidelity bipolar budding pattern typically observed in wild-type diploid cells, homozygous diploid pxl1s cells frequently budded at sites in the medial portion of the cell. As shown in Table 3, pxl1s/pxl1s cells initiated budding at aberrant sites $\sim 20$-fold more often than wild-type cells during their first two budding cycles and $\sim 10$-fold more frequently when the third budding cycle was also included in the analysis. These data suggest that pxl1s cells are compromised for a function associated with selecting or maintaining the location of polarized growth sites.

Polarized growth is also evident during mating, allowing yeast cells to respond to pheromone cues through the activation of cell surface receptors that direct cortical growth asymmetrically in the

Table 3. Diploid bud-site selection defects in pxl1 $\Delta / \mathrm{p} x 11 \Delta$ cells
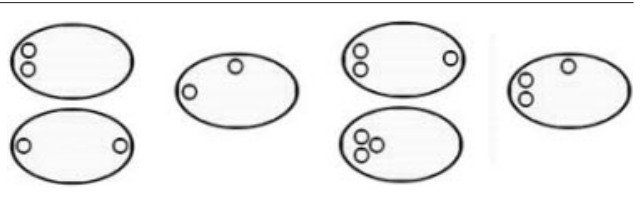

\begin{tabular}{lrrrr} 
& & & & \\
PXL11/PXL1 & 99.2 & 0.8 & 98.0 & 2.0 \\
\hline
\end{tabular}

Values represent the percentage of cells possessing the indicated bud-site selection patterns. 
$30^{\circ} \mathrm{C}$

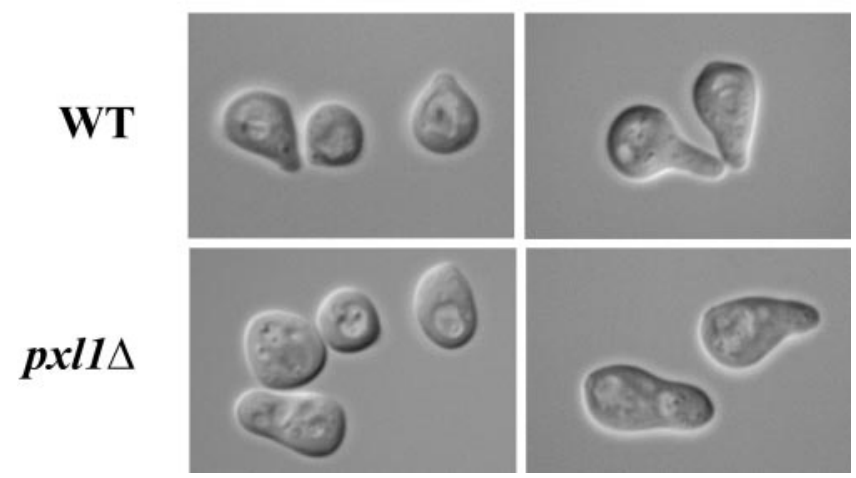

Figure 5. Haploid pxl1 $\Delta$ mutants possess defects in mating projection formation and morphology. At $30^{\circ}$, pxl1s cells form blunt mating projections compared with the narrow, focused projection tips characteristic of wild-type cells. Similarly, at $37^{\circ}$ wild-type cells form extended or hyperpolarized projections in contrast to the blunt projections formed by pxl1 $\Delta$ cells.

direction of the mating partner. To determine the effects of deleting PXL1 on polarized growth during mating, mating projection formation by haploid wild-type and pxl1 $\Delta$ cells was observed in mixtures of cells mating at either 30 or $37^{\circ} \mathrm{C}$. The morphology of mating projections produced by cells in mating mixtures is a sensitive indicator of cell polarization defects (Erdman et al., 1998). Normally, once a mating projection extends in length more than $\sim 0.5$ times the parent cell diameter, such projections are characterized by a focused tip. In contrast, we found that pxl1 $\Delta$ cells formed mating projections that exhibited broader, less focused, projections when compared with those of wild-type cells at both 30 and $37^{\circ} \mathrm{C}$ (Figure 5; Table 4). Interestingly, these polarization defects were especially prevalent at elevated temperatures for which the wild-type strain background polarizes more dramatically, suggesting a possible involvement of PXL1 in processes required for growth at high temperatures. These mating projection polarization defects did not, however, affect zygote formation or overall mating efficiency of $p x l 1 \Delta$ cells at any temperature (our unpublished results). Collectively, these data indicate that PXL1 is playing a role in polarized growth processes.

\section{PXL1 Displays Different Genetic Interactions with Both a GAP and an Effector of RHO1}

Activated Rho1p functions in the maintenance of cell polarity by signaling through its effectors: Pkclp, Fks1p, Bnilp,

Table 4. Mating projection morphology in wild type and pxl1s cells
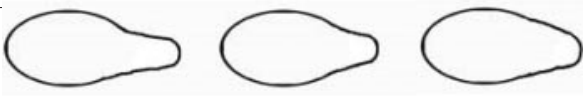

\begin{tabular}{|c|c|c|c|c|c|c|}
\hline & Hyperp & arized & & nal & & \\
\hline & $30^{\circ} \mathrm{C}$ & $37^{\circ} \mathrm{C}$ & $30^{\circ} \mathrm{C}$ & $37^{\circ} \mathrm{C}$ & $30^{\circ} \mathrm{C}$ & $37^{\circ} \mathrm{C}$ \\
\hline $\mathrm{T} \times \mathrm{UNT}$ & 1 & 49 & 56 & 25 & 43 & 26 \\
\hline$p x l 1 \Delta \times p x l 1 \Delta$ & 1 & 5 & 16 & 10 & 83 & 85 \\
\hline
\end{tabular}

Values represent the number of cells showing the morphology indicated at the respective temperature for $n=100$ cells scored.

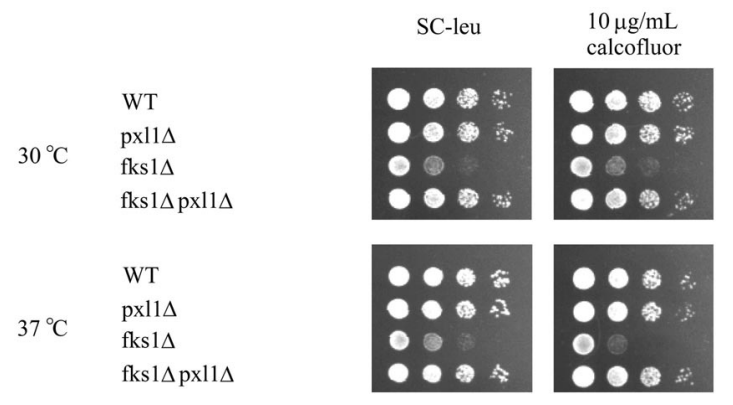

Figure 6. Removing PXL1 function suppresses the growth defect

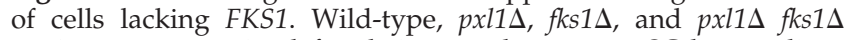
strains were examined for their growth rates on SC-leu medium containing $10 \mu \mathrm{g} / \mathrm{ml}$ calcofluor. Deletion of PXL1 can be seen to increase the growth rate of the $\operatorname{pxl1\Delta } f k s 1 \Delta$ relative to the $f k s 1 \Delta$ strain.

Sec $3 p$, and Skn7p. The effectors Fks1p, a catalytic subunit of the $\beta$-1,3-glucan synthase, and Skn7p, (Alberts et al., 1998; Ketela et al., 1999), are required for normal expansion of the cell wall during polarized growth (Qadota et al., 1992; Cabib et al., 1998; Watanabe et al., 2001; Sekiya-Kawasaki et al., 2002). Signaling through the effector Pkc1p, a homologue of the mammalian protein kinase $C$ family, regulates actin assembly, cell cycle progression, and expression of cell wall biosynthetic genes (Paravicini et al., 1992; Madden et al., 1997; Bickle et al., 1998; Delley and Hall, 1999). Additionally, Rho1p further modulates actin cytoskeletal organization and polarized secretion via interactions with Bnilp, a formin family member, and Sec3p, a member of the exocyst complex (Guo et al., 2001; Dong et al., 2003).

Because haploid pxl1s mutant cells exhibited defects in mating projection formation at elevated temperature, a condition in which the Rho1 pathway is active, and homozygous pxl1s mutant cells possessed bipolar budding pattern defects that further suggested a defect in the process of polarized growth, we constructed mutants between PXL1 and several nonessential genes mediating Rho1 pathway activities (i.e., BNI1, BCK1, and FKS1). We examined growth rates of the double mutant strains under conditions of Rho1 pathway activation: elevated temperature and on medium containing the cell wall and membrane destabilizing agents calcofluor white and SDS. Among the interactions tested, only in $p x l 1 \Delta f k s 1 \Delta$ mutant cells did we observe a phenotype different from the single mutants alone. Removal of PXL1 suppressed the calcofluor sensitivity caused by the loss of FKS1 as evidenced by the increased growth rate of the pxl1 $f k s 1 \Delta$ strain relative to the $f k s 1 \Delta$ strain (Figure 6). Additionally, we found both $f k s 1 \Delta$ and wild-type cells carrying a high copy plasmid encoding PXL1 grow at slightly reduced rates under these conditions (our unpublished results).

To further examine whether Pxl1p function is connected to the Rho1 pathway, we investigated whether Pxl1p interacted with components involved in GEF or GAP regulation of Rho1p. We constructed strains carrying null alleles of PXL1 and genes encoding specific GEFs or GAPs. Two GEFs are known to function to stimulate GDP-GTP exchange on Rho1p, Rom1p and Rom2p, among which Rom2p is the major activator (Manning et al., 1997; Bickle et al., 1998). Conversely, four GAPs: Bem2p, Sac7p, Lrg1p, and Bag7p are proposed to function to stimulate the intrinsic GTPase activity of Rho1p, downregulating its activities within the cell (Lorberg et al., 2001; Roumanie et al., 2001; Watanabe et al., 2001; Schmidt et al., 2002). Studies have suggested and sub- 
sequently shown that differential activities are mediated by these GAPs (Lorberg et al., 2001; Watanabe et al., 2001; Schmidt et al., 2002). Cells lacking both $L R G 1$ and SAC7 are inviable, whereas $\operatorname{lrg} 1 \Delta$ bem $2 \Delta$ and $\operatorname{sac} 7 \Delta$ bem $2 \Delta$ strains are viable for vegetative growth in other strain backgrounds and in the BY4743 background used for our studies (Lorberg et al., 2001; our unpublished results). We constructed bem $2 \Delta$ $p x l 1 \Delta, \operatorname{sac} 7 \Delta$ pxl1 $\Delta, \operatorname{lrg} 1 \Delta$ pxl1 , and rom $2 \Delta$ pxl1 $\Delta$ strains and compared their growth at different temperatures and in the presence of calcofluor white or SDS. As shown in Figure 7A, we observed no differences in growth between rom $2 \Delta$ pxl1 $\Delta$, sac7 $\operatorname{pxl1\Delta }$, and $\operatorname{lrg} 1 \Delta$ pxl1 $\Delta$ strains compared with rom $2 \Delta$, $\operatorname{sac} 7 \Delta$, or $\operatorname{lrg} 1 \Delta$ strains under any conditions tested. Strikingly, we did observe strong genetic interactions in strains deleted of both BEM2 and PXL1. Such strains displayed decreased ability to grow at temperatures other than $30^{\circ} \mathrm{C}$ and hypersensitivity to SDS under conditions of elevated temperature. These effects are specific to the GAP Bem $2 p$, because loss of PXL1 activity has no effect in strains lacking the GAPs Lrg1p or Sac7p, nor the GAPs for Cdc42p, Rga1p, and Bem $3 p$ (our unpublished results).

The compromised growth rate observed for the bem $2 \Delta$ pxl1s strain suggested that such cells are likely to possess defects in cell morphology, controlled deposition of cell wall components, and therefore cell integrity. We examined these phenotypes by staining cells with the vital dye methylene blue that is excluded from viable cells but specifically stains dead cells. Table 5 presents the results of quantifying the fraction of viable cells in cultures of these strains grown at different temperatures. These data clearly show that bem $2 \Delta$ pxl1 $\Delta$ cells are less viable than wild-type, or $p x l 1 \Delta$ or bem $2 \Delta$ strains, particularly under conditions that require activity of Rho1 signaling and the cell integrity MAP kinase pathway. In addition, although many bem $2 \Delta$ cells appear enlarged relative to wild-type cells, the bem $2 \Delta$ pxl1 $\Delta$ cells appeared still more aberrant in morphology. Principally these cells displayed a greater increase in cell size and the overall fraction of enlarged cells; a similar fraction of bem $2 \Delta$ pxl1 $\Delta$ cells displayed the multiple small, irregularities observed in bem $2 \Delta$ cells (Figure 7B). Furthermore, the high-temperature growth defect of bem $2 \Delta$ pxl1s cells was not sorbitol remedial, in contrast to bem $2 \Delta$ cells (our unpublished results).

To help ascertain if the polarity defects caused by a lack of Pxl1p were restricted to a function associated with Rho1p dependent activities or whether the functions of other Rho GTPases such as Cdc42p might be involved, we constructed strains containing replacements of PXL1 and nonessential effectors and regulators of the Cdc42 pathway. We observed no synthetic genetic interactions between loss of PXL1 function and the loss of $\mathrm{Cdc} 42$ pathway members, including BEM3, RGA1, RGA2, GIC1, GIC2, STE20, and CLA4. Similarly, we observed no synthetic genetic interactions between the loss of PXL1 and the removal of RHO2, another small GTPase that plays a similar but minor role relative to $\mathrm{RHO1}$ during vegetative growth. Together these data indicate that the loss of PXL1 has distinct effects on Rho1 signaling and pathway function, particularly in strains also missing the function of the Rho GAP, Bem2p, and the effector Fks1p.

\section{Individual LIM Domains Play Essential and Unique Roles in Pxl1p Localization and Function}

It has been previously shown that within paxillin, the LIM domains required for its targeting each possess different functional specificities. LIM3 plays a major role in targeting paxillin to focal adhesions, whereas LIM2 plays a supporting role in combination with LIM3 for polarized growth site targeting (Brown et al., 1996). The proteins bound by these domains that mediate their potential functions in focal complex organization and signaling largely remain to be determined. To investigate whether the LIM domains of Pxl1p play distinct roles in polarized growth site targeting, we used site-directed mutagenesis to disrupt residues within each of the individual LIM domains in the GFP::Pxl1p. Mutagenesis studies of paxillin LIM3 previously showed that substitution of conserved histidine and cysteine residues required for $\mathrm{Zn}^{2+}$ coordination within the LIM domain with isoleucine and alanine residues, respectively, abolished focal contact localization. We introduced mutations into the codons for the cognate residues within LIM1 of GFP::Pxl1p-H581I and C584A. Similar changes were made in a separate construct within LIM2: H642I and C645A. These mutations are predicted to eliminate the coordination of one zinc ion within each finger of the LIM domains, presumably prohibiting their proper folding and subsequent interactions with LIM-binding partner proteins.

To examine how interfering with the structures of the individual LIM domains of Pxl1p affected the ability of Pxl1p to function in cells lacking Bem2p, we evaluated the abilities of plasmid encoded full-length Pxl1 proteins carrying either the LIM1 (H581I/C584A) or LIM2 (H642I/C645A) mutations (denoted pxl1-lim1 and pxl1-lim2) to complement the growth defects of bem $2 \Delta$ pxl1s strains under different conditions. As shown in Figure 8A, these forms of Pxl1p lacking either LIM domain were unable to complement the bem $2 \Delta$ pxl1s growth defect. The viabilities of bem $2 \Delta$ pxl1s cells carrying the pxl-lim1 and pxl-lim2 mutants also was not significantly different from the same strain carrying an empty vector control as assessed using the vital dye methylene blue (empty vector $24 \%$ viable, pxl1-lim $128 \%$ viable, pxl1-lim2 36\% viable, as compared with wild-type PXL1 $73 \%$ viable; $n=200$ cells examined). These data therefore indicate that both LIM domains are essential for normal function of Pxl1p.

To further explore how the individual LIM domains may be required for Pxllp function, we examined their roles in protein targeting. Localization patterns of the GFP::pxl1-limH581I/C584A and GFP::pxl1-limH642I/C645A fusion proteins were therefore examined in a $p x l 1 \Delta$ strain. As shown in Figure 8B, inactivation of the Pxl1p LIM1 or LIM2 domains created strikingly distinct affects on the localization of Pxl1p. Elimination of the Pxl1p LIM1 domain function abolished bud tip localization but not mother-bud neck localization of the fusion protein, although the frequency of cells displaying the latter localization was reduced approximately twofold. Moreover, the amount of Pxl1p that appeared to reside at mother-bud neck localization sites was similar for the LIM1 mutant to that observed with the wild-type GFP-Pxl1p. In contrast, mutation of the Pxl1p LIM2 domain abolished localization to mother-bud neck sites completely but also very strongly reduced the frequency of localization to bud sites. Additionally, the quantity of GFP-Pxl1p residing at these sites in cells displaying bud localizations appeared to be substantially reduced in amount based on the relative intensity of the GFP signal. These data therefore indicate that the individual LIM domains each play essential and unique roles in the localization of Pxl1p.

In summary, the inabilities of Pxl1 proteins separately lacking the function of either the LIM1 or LIM2 domain to rescue the growth defects of the bem $2 \Delta$ pxl1 $1 \Delta$ strain and to localize to both bud sites and sites of cytokinesis indicates that the collective, and presumably sequential, functions of these LIM domains are each essential. The requirement for both LIM domains of Pxl1p to accomplish its correct localization and growth functions strongly suggests that it is likely to perform polarized growth functions in both grow- 
A

\begin{tabular}{|c|c|c|c|c|c|c|}
\hline & & YPAD & $\begin{array}{l}0.005 \% \\
\text { SDS }\end{array}$ & $\begin{array}{l}0.01 \% \\
\text { SDS }\end{array}$ & $\begin{array}{c}5 \mu \mathrm{g} / \mathrm{mL} \\
\text { calcofluor }\end{array}$ & $\begin{array}{l}10 \mu \mathrm{g} / \mathrm{mL} \\
\text { calcofluor }\end{array}$ \\
\hline $25^{\circ} \mathrm{C}$ & $\begin{array}{l}\text { WT } \\
\text { pxl1 } \Delta \\
\text { rom2 } 2 \Delta \\
\text { bem2 } 2 \Delta \\
\operatorname{sac7\Delta } \\
\operatorname{lrg} 1 \Delta \\
\operatorname{rom} 2 \Delta \text { pxl1 } \Delta \\
\text { bem2 } 2 \Delta \text { pxl1 } \\
\operatorname{sac7\Delta } \text { pxl1 } \\
\operatorname{lrg} 1 \Delta \text { pxl1 }\end{array}$ & 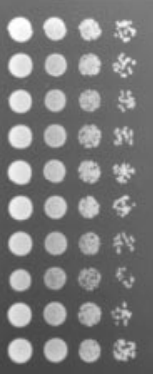 & 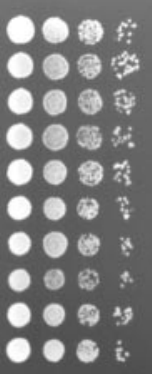 & 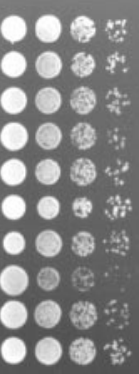 & $\begin{array}{l}00 \\
00 \\
0 \\
0 \\
0\end{array}$ & 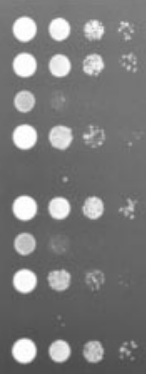 \\
\hline $30{ }^{\circ} \mathrm{C}$ & $\begin{array}{l}\text { WT } \\
\text { pxl1 } \Delta \\
\text { rom2 } 2 \Delta \\
\text { bem2 } 2 \Delta \\
\operatorname{sac} 7 \Delta \\
\operatorname{lrg} 1 \Delta \\
\operatorname{rom} 2 \Delta \text { pxl1 } \\
\text { bem2 } 2 \Delta \text { px11 } \\
\operatorname{sac} 7 \Delta \text { pxl1 } \\
\operatorname{lrg} 1 \Delta \text { pxl1 }\end{array}$ & 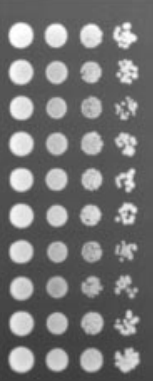 & 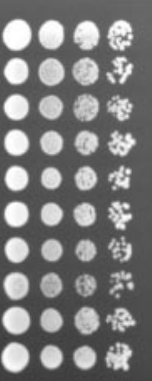 & 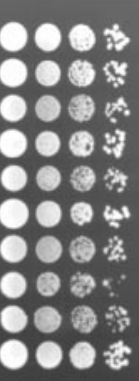 & 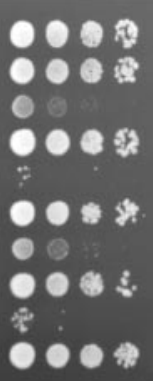 & 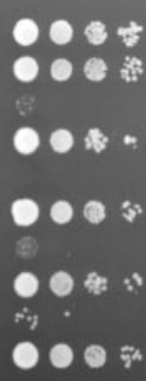 \\
\hline $37^{\circ} \mathrm{C}$ & $\begin{array}{l}\text { WT } \\
\text { pxl1 } \Delta \\
\text { rom2 } 2 \Delta \\
\text { bem2 } 2 \Delta \\
\operatorname{sac} 7 \Delta \\
\operatorname{lrg} 1 \Delta \\
\operatorname{rom} 2 \Delta \text { pxl1 } \\
\text { bem } 2 \Delta \text { pxl1 } \\
\operatorname{sac} 7 \Delta \text { pxl1 } \\
\operatorname{lrg} 1 \Delta \text { pxl1 }\end{array}$ & 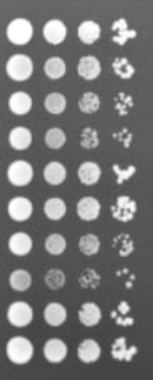 & 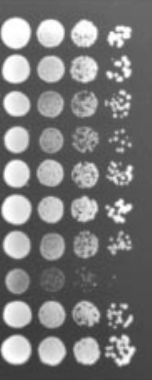 & 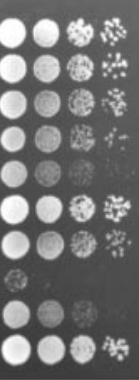 & $\begin{array}{l}90 \% \\
005 \\
0 \\
0 \\
0 \\
0 \\
0 \\
0 \\
0\end{array}$ & $\stackrel{0}{\circ}$ \\
\hline
\end{tabular}

B
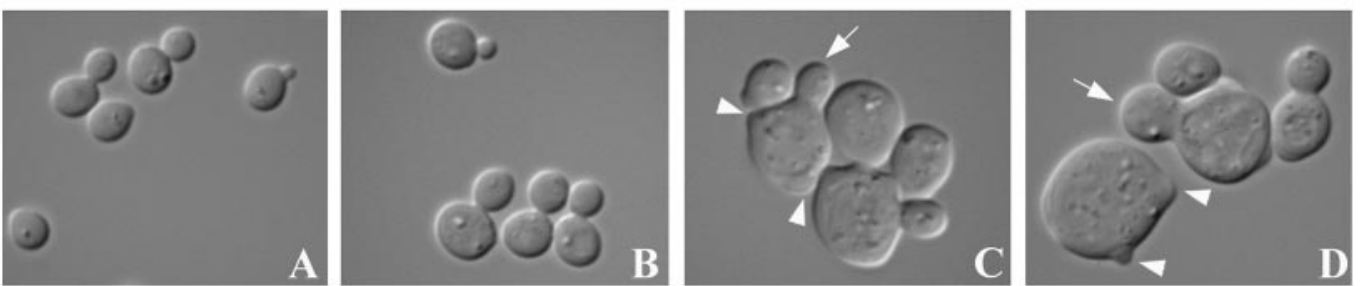

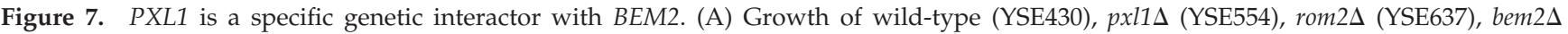
(YSE661), sac7 $($ YSE818), and lrg1 (YSE804) strains was compared with double mutants lacking both PXL1 and one of the RHO1 GEF or

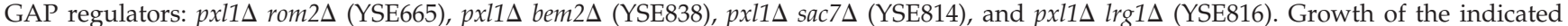
strains was examined at 25,30 , and $37^{\circ} \mathrm{C}$ on YPAD medium or YPAD supplemented with the cell wall destabilizing agents calcofluor white or SDS as indicated. Removal of PXL1 function clearly affects pxl1 bem $2 \Delta$ relative to bem $2 \Delta$ cells under conditions of elevated temperature or the presence of SDS (Rho1-activating conditions). This genetic interaction is specific to BEM2 loss, as it is not observed with the other Rho1p regulators examined. (B) Morphologies of wild-type, pxl1s, bem $2 \Delta$, and bem2 $2 x$ pl1 $\Delta$ were compared using DIC microscopy. Relative to wild-type (A) and pxl1 $($ B) cells, both bem $2 \Delta$ (C) and bem $2 \Delta$ pxl1 $(\mathrm{D})$ cells appear enlarged (arrows) and often display multiple buds per cell (arrowheads). Comparison of bem $2 \Delta$ cells to bem $2 \Delta$ pxl1 $1 \Delta$ cells revealed bem $2 \Delta$ pxl1 $\Delta$ cells to be larger, frequently possessing wider bud necks. 
Table 5. Viability of wild type, bem $2 \Delta$, and bem $2 \Delta$ pxl1 $1 \Delta$ cells

\begin{tabular}{|c|c|c|c|c|}
\hline & $25^{\circ} \mathrm{C}$ & $30^{\circ} \mathrm{C}$ & $37^{\circ} \mathrm{C}$ & $39^{\circ} \mathrm{C}$ \\
\hline WT & $99.7(0.3)$ & $99.8(0.2)$ & $99.7(0.2)$ & $100.0(0.0)$ \\
\hline pxl1s & $99.2(0.3)$ & $99.2(0.2)$ & $99.7(0.2)$ & $99.3(0.2)$ \\
\hline bem $2 \Delta$ & $97.0(1.5)$ & $96.7(0.4)$ & $95.5(2.3)$ & $93.7(0.2)$ \\
\hline bem $2 \Delta$ pxl1s & $95.2(1.4)$ & $94.5(1.0)$ & $86.8(0.7)$ & $79.3(1.9)$ \\
\hline
\end{tabular}

Viability is expressed as the percentage of methylene-blue-negative cells scored at the different temperatures for three independent experiments. Standard error is indicated in parentheses.

ing buds and at the mother bud neck late in the cell cycle, consistent with its localization to these sites.

\section{Localization and Maintenance of Pxl1p at Polarized Growth Sites}

We next examined the requirements of Pxl1p localization to polarized growth sites for several different pathways and components that have been previously shown to play roles in localizing cell polarity proteins. Previous work using the actin-depolymerizing drug latrunculin has demonstrated a number of proteins, including: Abp1p, Cofilin, Sla1p, Sla2p, and Sec4p as well as Sec8p, require an intact actin cytoskeleton for their correct delivery to polarized growth sites (Ayscough et al., 1997; Ayscough, 2000). In addition to being delivered to growth sites by the actin cytoskeleton, a pool of the protein Aip3/Bud6p, and more recently other polarized proteins such as a regulatory subunit of the protein phosphatase $2 \mathrm{~A}$, have been shown to localize to growth sites

A

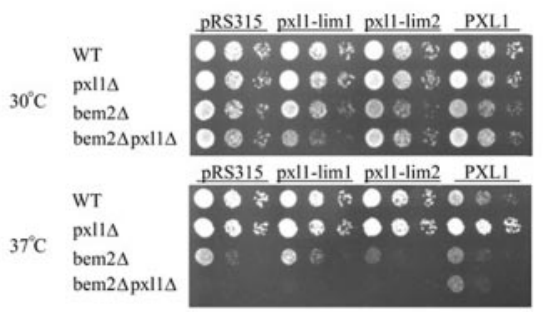

B

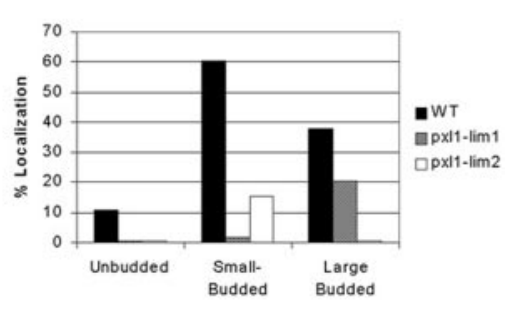

Figure 8. The LIM1 and LIM2 domains of Pxl1p are each required for its function and play distinct roles in Pxl1p subcellular localization. (A) Functional analysis of PXL1-H581I/C584A (pxl1-lim1) and PXL1-H642I/C645A (pxl1-lim2) mutants by complementation of bem $2 \Delta$ pxl1s growth defects. Neither of these mutants is able to complement loss of PXL1 function. (B) Localization to polarized sites of GFP::Pxl1-H581I/C584A and GFP::Px11-H642I/C645A mutants in pxl1s cells reveals unique requirements for each LIM domain in growth site localizations.
Table 6. GFP::Pxl1p localization is independent of the secretory pathway

\begin{tabular}{|c|c|c|c|c|c|c|}
\hline & \multicolumn{6}{|c|}{ Localization $^{\mathrm{a}}$} \\
\hline & \multicolumn{2}{|c|}{ Unbudded } & \multicolumn{2}{|c|}{ Small-budded } & \multicolumn{2}{|c|}{$\begin{array}{l}\text { Medium-large } \\
\text { budded }\end{array}$} \\
\hline & $25^{\circ} \mathrm{C}$ & $37^{\circ} \mathrm{C}$ & $25^{\circ} \mathrm{C}$ & $37^{\circ} \mathrm{C}$ & $25^{\circ} \mathrm{C}$ & $37^{\circ} \mathrm{C}$ \\
\hline WT & 7.5 & 3.9 & 69.0 & 52.9 & 20.3 & 13.5 \\
\hline $\sec 17-1$ & 10.6 & 10.9 & 33.3 & 24.1 & 23.4 & 11.2 \\
\hline $\sec 14-3$ & 13.3 & 21.1 & 53.3 & 68.6 & 22.7 & 8.5 \\
\hline $\sec 4-8$ & 21.0 & 16.2 & 76.2 & 46.7 & 24.0 & 12.2 \\
\hline
\end{tabular}

a Values represent the percentage of cells displaying the wild type GFP ::Pxl1p localization pattern at the indicated temperature $(\mathrm{n}=$ 200).

through a function of the late secretory pathway (Jin and Amberg, 2000; Gentry and Hallberg, 2002). Finally, the functional integrity of the septins has been shown to be necessary to maintain the localization of a variety of bud-neck associated kinases such as Gin $4 p$ and Kcc4p as well as the proteins associated with them.

We examined the frequency of cells displaying GFP::Pxl1p localization at each of the different polarized growth sites at which it is found (incipient bud sites, growing buds, and mother-bud neck) in cells in which the activities of each of these systems were inactivated either by drug treatment (latrunculin B) or by raising strains to a restrictive temperature (sec mutants; $c d c 12-6^{t s}$ allele). As indicated by Table 6 , we found Pxl1p localization to be effectively independent of secretory pathway function for periods of at least $90 \mathrm{~min}$ after a shift to the restrictive temperature. We also examined Pxl1p localization dependence on septin function in $c d c 12-$ $6^{\text {ts }}$ cells shifted to their restrictive temperature of $37^{\circ} \mathrm{C}$ during which the septins are known to disassemble in such cells. Unfortunately, the incomplete septation of these cells substantially complicated an extensive quantification of GFP::Pxl1p localization to different polarized growth sites. We did observe Pxl1p to remain localized at bud tips and at the mother-bud neck in cells after the temperature shift for 90 min, suggesting that maintenance of Pxl1p localization at growth sites is largely independent of septin integrity. We also investigated the dependence of Pxl1p localization on an intact actin cytoskeleton. As shown in Table 7, the maintenance of Pxl1p at polarized growth sites is substantially dependent on the integrity of F actin structures. This actin dependence is in contrast to Aip3/Bud6p localization, which is stable to actin depolymerization once the protein is delivered to polarized growth sites and only partially dependent on actin for its initial delivery (Ayscough et al., 1997; Jin and Amberg, 2000; D. Amberg, personal communications). Thus, it appears that Pxl1p and Aip3p are maintained at polarized growth sites through different sets of proteins, with those involved in Pxllp localization being dependent on an intact actin cytoskeleton.

\section{DISCUSSION}

Growth Site Targeting Is a Common and Ancient Function of LIM Domains in Eukaryotes

An important goal for understanding polarized growth mechanisms is to determine the full complement of proteins 
Table 7. Maintenance of GFP ::Pxl1p localization is dependent on the actin cytoskeleton

\begin{tabular}{|c|c|c|c|c|c|c|c|c|c|c|c|c|c|}
\hline & \multirow[b]{3}{*}{ Lat-B } & \multicolumn{12}{|c|}{ Localization $^{\mathrm{a}}$} \\
\hline & & \multicolumn{4}{|c|}{ Unbudded } & \multicolumn{4}{|c|}{ Small-budded } & \multicolumn{4}{|c|}{ Medium-large budded } \\
\hline & & 0 & 30 & 60 & 90 & 0 & 30 & 60 & 90 & 0 & 30 & 60 & 90 \\
\hline GFP::Pxl1p & - & 10.2 & 17.3 & 8.5 & 15.2 & 53.1 & 37.9 & 28.8 & 41.2 & 9.5 & 22.7 & 27.4 & 23.8 \\
\hline GFP $:: P x 11 p$ & + & 2.5 & 3.5 & 3.1 & 0.0 & 25.8 & 0.0 & 5.9 & 4.8 & 13.6 & 12.5 & 23.5 & 14.3 \\
\hline GFP::Aip3p & - & 65.0 & nd & nd & 39.1 & 95.6 & nd & nd & 100.0 & 95.7 & nd & nd & 90.5 \\
\hline GFP :: Aip3p & + & 70.0 & nd & nd & 51.3 & 100.0 & nd & nd & 100.0 & 94.0 & nd & nd & 92.4 \\
\hline
\end{tabular}

a Values represent the percentage of cells displaying the wild type GFP::Pxl1p localization pattern out of the total number of cells examined at each time point $(n=200)$. nd, not determined.

acting at such sites as well as their dynamics of assembly and disassembly in regard to growth signals and/or cell cycle cues. During polarized growth, dynamic reformation of the local actin cytoskeleton occurs in response to localized signaling via the complement of polarity proteins and local activation of small GTPases such as Ras, Rac, Rho, and Cdc42 occurring at these sites. In metazoan cells, an important class of these sites at which actin is concentrated are termed focal contacts. The assembly of such sites is mediated by a key scaffolding protein, paxillin, a group 3 LIM domain protein. Analogous structures to focal contacts exist in simpler eukaryotes, such as Dictyostelium discoidium and S. cerevisiae. These structures are defined by cortical actin patches and similarly play a role in growth targeting. Although a paxillin homologue has yet to be identified in either of these simpler eukaryotes, group 3 LIM domain proteins are present in both organisms that bear homology to specific LIM domains of paxillin and participate in polarized growth processes. For example, in D. discoidium, limB/LIM2 is required for proper organization of the actin cytoskeleton and is subsequently involved in such processes as morphogenesis, including tip formation (Chien et al., 2000). Similarly in S. cerevisiae, as we have shown here, Pxl1p is a component of polarized growth sites and is required for proper bud-site selection in diploid cells and polarized growth during mating. These processes are known to require dynamic reorganization of the yeast actin cytoskeleton (Herskowitz et al., 1995; Pringle et al., 1995; Chant, 1999; Casamayor and Snyder, 2002).

The general role of Group 3 LIM domain proteins as modulators of the actin cytoskeleton at polarized growth sites, whether during polarity establishment or maintenance, suggests members of this subgroup of LIM family proteins share an evolutionary conserved function among the varied activities of different classes of LIM domain proteins that operate to control cellular differentiation. Our studies lend confirmation to this idea by demonstrating a role in the modulation of small GTPase signaling and polarized growth for Pxl1p, one of the four LIM proteins encoded by the budding yeast genome. Other classes of LIM domain containing proteins, most notably nuclear proteins participating principally in transcriptional regulation such as the canonical LIM homeodomain (LHX), LIM only (LMO), and LIM kinase group 1 LIM proteins are completely absent from the budding yeast genome and have yet to be identified in the slime mold. Therefore, the cytosolic function of coordinating signal transduction and cytoskeletal regulation at cortical sites among these LIM domain-containing proteins appears to represent a primitive function in the development of eucaryotic cell structure.
Additionally, we have shown each of the LIM domains of Pxl1p to perform specific and essential roles for targeting of the protein to sites of polarized cell growth. Such specialization of LIM domain function is not uncommon: the individual LIM domains of paxillin have each been reported to mediate distinct protein interactions and localization activities (Brown et al., 1996, 1998; Brown and Turner, 1999), the LIM domains of Rga1/Dbm1p in S. cerevisiae are uniquely required for function (Chen et al., 1996), and analyses of slime mold group 3 LIM proteins such as limB/LIM2 and LimC have revealed specific roles for individual LIM domains within their respective proteins (Prassler et al., 1998; Chien et al., 2000; Khurana et al., 2002a). At present, how specificity of interaction is conferred within this conserved protein-protein interaction motif to control which partner proteins are bound by distinct LIM domains remains to be elucidated.

\section{Pxl1p Is a Likely Modulator of Rho GTPase-mediated Signaling at Yeast Growth Sites}

Reorganization of the actin cytoskeleton via signaling through the small Rho GTPases encoded by CDC42 and RHO1 is required during budding in diploid cells and during mating projection formation in response to pheromone Our studies demonstrate the involvement of Pxl1p in cell polarity functions in budding yeast (Tables 3-5). The inability of pxl1 mutant cells to polarize at high temperature in response to mating pheromone and our current knowledge concerning its genetic interactions with loci affecting Rho1 pathway activities (Figures 6 and 7), suggest a potential role for Pxl1p as a modulator of small GTPase signaling. Such a function is consistent with the localization of Pxl1p to polarized growth sites in a distribution that is highly similar to that reported for the activated GTP bound form of Rho1p as well as selected GEF and GAP regulators such as Rom $2 p$ and Lrg1p (Drgonova et al., 1996; Manning et al., 1997; Watanabe et al., 2001).

Genetic interactions of PXL1 with the Rho1p GAP encoded by BEM2 and the Rho1p effector and $\beta-1,3$ glucan synthase complex subunit encoded by FKS1 are consistent with its functioning in the control of yeast cell polarity. Based on our genetic studies, however, it is impossible to further conclude how Pxl1p is acting at the molecular level. The opposing genetic interactions with BEM2 and FKS1 and the requirement of PXL1 for normal mating polarization at elevated temperature support a Rho pathway-modulating activity of Pxl1p. It is also interesting in this regard that the three remaining LIM domain-containing proteins encoded by the yeast genome: Rga1p, Rga2p, and Lrg1p, each play 
negative regulatory roles in small GTPase signaling (Stevenson et al., 1995; Roumanie et al., 2001; Smith et al., 2002).

That loss of PXL1 exacerbates a bem $2 \Delta$ phenotype does suggest Pxl1p to perhaps play a negative, inhibitory, role in restricting Rho1p interactions and activities. Comparison of bem $2 \Delta$ and bem $2 \Delta$ pxl1s cell morphologies revealed a size increase in bem $2 \Delta$ pxl1 $\Delta$ cells, an effect that has been associated with hyperactivated Rho1p in cells (Wang and Bretscher, 1995). The additive effects of loss of Pxl1p function in bem $2 \Delta$ cells are particularly notable at elevated temperatures and in the presence of SDS, conditions under which Bem $2 p$ has been shown to regulate the $P k c 1$ pathway is active in regulating actin organization (Delley and Hall, 1999; Martin et al., 2000). The suppression of $f k s 1 \Delta$ defects in fks $1 \Delta$ pxl1 $1 \Delta$ cells, an effect opposite to that occurring in the absence of BEM2, provides further evidence for a proposed negative regulatory function of Pxl1p. Removing Pxl1p function may cause such an effect either by creating a general increase in Rholp activities on parallel effectors such as: Pkc1p, Sec3p, or Skn7p, or by removing a specific negative regulatory activity for the effector Fks1p (and presumably Fks2p, which would remain active in $f k s 1 \Delta$ cells). Previous studies have shown increased signaling through the cell integrity pathway to induce Fks2p expression in the absence of FKS1 (Zhao et al., 1998). Furthermore, such a negative regulatory role is supported by observations by both our group and others that overexpression of Pxl1p enhances the growth defects of $r h o 1^{t s}$ but not $c d c 42^{t s}$ strains when grown at semipermissive temperatures (our unpublished results; E. $\mathrm{Bi}$, personal communication). However, redundancies among the different Rho GAPs and interactions between the Rho1 and Cdc42 GAPs and effector pathways make it difficult to further interpret the causes of these synthetic genetic interactions with PXL1.

Although our data suggest a Rho1 pathway-directed activity of Pxl1p, given the relatively strong growth phenotypes of mutants in both BEM2 and FKS1, other roles for Pxl1p clearly remain possible. BEM2 interacts genetically with many cell polarity components and regulators, including MYO1, MYO2, TPM1, SAC6, MSB1, BEM1, ACT1, and RHO1 (Bender and Pringle, 1991; Peterson et al., 1994; Wang and Bretscher, 1995). Additional studies linking Bem2p functions to other growth-regulating activities and the Cdc42 pathway suggest alternative, Rho1 pathway-independent targets of Pxl1p function. Presently, there is evidence for a GAP-independent role for Bem2 $\mathrm{p}$ in a morphogenesis checkpoint (Marquitz et al., 2002). Also, certain alleles of the Cdc42 pathway GAP Rga1p can rescue the morphology defects in bem $2 \Delta$ mutant cells, and recent in vitro evidence has demonstrated Bem $2 p$ to have GAP activity on Cdc42p (Chen et al., 1996; Marquitz et al., 2002). Thus, it is conceivable that Pxl1p might participate in regulating Cdc42p or one of its effectors.

As studies in mammalian cells have demonstrated, the mechanisms involved in polarity establishment (Rac and Cdc42) and those involved in polarity maintenance (Rho1) are closely networked. To coordinate polarized growth, these mechanisms may use feedback loops upon one another, making genetic dissections of the pathways challenging. At present, although we cannot exclude the possibility that Pxl1p acts on other Rho GTPases in yeast or in other cell polarity functions that more peripherally related to Rho1 pathway functions, we consider these possibilities to be less likely for several reasons. First, Pxl1p overexpression strongly enhances the severity of growth defects of rho1 ${ }^{\text {ts }}$ alleles and not $c d c 42^{t s}$ strains when grown at semipermissive temperatures (our unpublished results; E. Bi, personal com- munication). Second, recent studies indicate the ability of Pxl1p to directly interact with Rho1p in vitro, and this interaction again does not occur with Cdc42p (X.-D. Gao and E. Bi, personal communication). Finally, several of the abovementioned activities of the Rga1 and Bem 2 proteins were observed with truncated versions of them that were largely limited to their GAP domains; such proteins may lack elements that normally confer specificity to one RhoGTPase or another. Consistent with this, the full-length Rga1p is unable to rescue bem $2 \Delta$ defects, and conflicting evidence exists as to whether or not Bem $2 p$ GAP domain is able to provide GAP activity in the presence of yeast Cdc42p (Zheng et al., 1994; Chen et al., 1996). Thus, there is the potential that these activities may not reflect the normal in vivo roles of these proteins, and we view the genetic data we present as most consistent with a Rho1 pathway-modulating activity of Pxl1p.

Our studies here establish Pxl1p as a cell polarity protein in yeast that may function similarly to mammalian paxillin, as a scaffolding protein that aids in organizing and modulating the activities of signaling and microfilament cytoskeleton reorganizing components. Pxl1p is targeted to polarized growth sites: bud tip and mother-bud neck, primarily through the independent activities of its LIM domains, and its role with respect to Bem $2 p$ function is required in both contexts, suggesting a common function in polarity at such sites. Its specific function(s) in protein complexes present at growth sites, and its exact role(s) in small GTPase signaling remain to be understood. Further analyses of the targeting and complex assembly mediated by Pxl1p during polarized growth events in yeast, via analyses of the LIM domain contribution to specificity, may provide insight into the mechanisms for the organization of metazoan paxillin family proteins. Such information should help define general and conserved mechanisms, analogous and homologous, of cell polarity regulation in eukaryotes.

\section{ACKNOWLEDGMENTS}

We thank Charlie Boone, Michael Hall, David Amberg, and the Yeast Resource Center for gifts of plasmids and strains helpful to these studies. We are also grateful to David Amberg, Brian Haarer, and Chris Turner for many valuable discussions and Xiang-Dong Gao and Erfei Bi for communications about their work on PXL1 before publication. This work was supported in part by funding from the Syracuse University Ruth Meyer Undergraduate Scholars Fund to T.J.S. and an National Science Foundation Grant MCB0131408 to S.E.E.

\section{REFERENCES}

Adams, A.E.M., Johnson, D.I., Longnecker, R.M., Sloat, B.F., and Pringle, J.R (1990). CDC42 and CDC43, two additional genes involved in budding and the establishment of cell polarity in the yeast Saccharomyces cerevisiae. J. Cell Biol. $111,131-142$

Alberts, A.S., Bouquin, N., Johnston, L.H., and Treisman, R. (1998). Analysis of RhoA-binding proteins reveals an interaction domain conserved in heterotrimeric $G$ protein beta subunits and the yeast response regulator protein Skn7. J. Biol. Chem. 273, 8616-8622.

Altschul, S.F., Gish, W., Miller, W., Meyers, E.W., and Lipman, D.J. (1990). Basic local alignment search tool. J. Mol. Biol. 215, 403-410.

Arkowitz, R.A. (1999). Responding to attraction: chemotaxis and chemotropism in Dictyostelium and yeast. Trends Cell Biol. 9, 20-27.

Ayscough, K.R. (2000). Endocytosis and the development of cell polarity in yeast require a dynamic F-actin cytoskeleton. Curr. Biol. 10, 1587-1590.

Ayscough, K.R., Stryker, J., Pokala, N., Sanders, M., Crews, P., and Drubin, D.G. (1997). High rates of actin filament turnover in budding yeast and roles for actin in establishment and maintenance of cell polarity revealed using the actin inhibitor latrunculin-A. J. Cell Biol. 137, 399-416.

Bach, I. (2000). The LIM domain: regulation by association. Mech. Dev. 91, $5-17$. 
Baudin, A., Ozier-Kalogeropoulos, O., Denouel, A., Lacroute, F., and Cullin, C. (1993). A simple and efficient method for direct gene deletion in Saccharomyces cerevisiae. Nucleic Acids Res. 21, 3329-3330.

Bender, A., and Pringle, J.R. (1991). Use of a screen for synthetic lethal and multicopy suppressee mutants to identify two new genes involved in morphogenesis in Saccharomyces cerevisiae. Mol. Cell. Biol. 11, 1295-1305.

Bickle, M., Delley, P.A., Schmidt, A., and Hall, M.N. (1998). Cell wall integrity modulates RHO1 activity via the exchange factor ROM2. EMBO J. 8, 22352245.

Bretscher, A. (2003). Polarized growth and organelle segregation in yeast: the tracks, motors, and receptors. J. Cell Biol. 160, 811-816.

Brown, M.C., Perrotta, J.A., and Turner, C.E. (1996). Identification of LIM3 as the principal determinant of paxillin focal adhesion localization and characterization of a novel motif on paxillin directing vinculin and focal adhesion kinase binding. J. Cell Biol. 135, 1109-1123.

Brown, M.C., Perrotta, J.A., and Turner, C.E. (1998). Serine and threonine phosphorylation of the paxillin LIM domains regulates paxillin focal adhesion localization and cell adhesion to fibronectin. Mol. Biol. Cell 9, 1803-1816.

Brown, M.C., and Turner, C.E. (1999). Characterization of paxillin LIM domain-associated serine threonine kinases: activation by angiotensin II in vascular smooth muscle cells. J. Cell. Biochem. 76, 99-108.

Burns, N., Grimwade, B., Ross-Macdonald, P.B., Choi, E.-Y., Finberg, K., Roeder, G.S., and Snyder, M. (1994). Large-scale characterization of gene expression, protein localization and gene disruption in Saccharomyces cerevisiae. Genes Dev. 8, 1087-1105.

Cabib, E., Drgonova, J., and Drgon, T. (1998). Role of small G proteins in yeast cell polarization and wall biosynthesis. Annu. Rev. Biochem. 67, 307-333.

Casamayor, A., and Snyder, M. (2002). Bud-site selection and cell polarity in budding yeast. Curr. Opin. Microbiol. 5, 179-186.

Chant, J. (1999). Cell polarity in yeast. Annu. Rev. Cell Dev. Biol. 15, 365-391.

Chant, J., and Stowers, L. (1995). GTPase cascades choreographing cellular behavior: movement, morphogenesis and more. Cell 81, 1-4.

Chen, D.-C., Yang, B.-C., and Kuo, T.-T. (1992). One step-transformation of yeast in stationary phase. Curr. Genet. 21, 83-84.

Chen, G., Zheng, L., and Chan, C.S.M. (1996). The LIM domain-containing Dbm1 GTPase-activating protein is required for normal cellular morphogenesis in Saccharomyces cerevisiae. Mol. Cell. Biol. 16, 1376-1390.

Chien, S., Chung, C.Y., Sukumaran, S., Osborne, N., Lee, S., Ellsworth, C., McNally, J.G., and Firtel, R.A. (2000). The Dictyostelium LIM domain-containing protein LIM2 is essential for proper chemotaxis and morphogenesis. Mol. Biol. Cell 11, 1275-1291.

Christianson, T.W., Sikorski, R.S., Dante, M., Shero, J.H., and Hieter, P. (1992). Multifunctional yeast high-copy-number shuttle vectors. Gene 110, 119-122.

Cliften, P., Sudarsanam, P., Desikan, A., Fulton, L., Fulton, B., Majors, J., Waterston, R., Cohen, B.A., and Johnston, M. (2003). Finding functional features in Saccharomyces genomes by phylogenetic footprinting. Science $301,71-76$

Delley, P.A., and Hall, M.N. (1999). Cell wall stress depolarizes cell growth via hyperactivation of RHO1. J. Cell Biol. 147, 163-174.

Dong, Y., Pruyne, D., and Bretscher, A. (2003). Formin-dependent actin assembly is regulated by distinct modes of Rho signaling in yeast. J. Cell Biol. 161, 1081-1092.

Dorer, R., Boone, C., Kimbrough, T., Kim, J., and L.H. Hartwell, L.H. (1997). Genetic analysis of default mating behavior in Saccharomyces cerevisiae. Genetics $146,39-55$.

Drgonova, J., Drgon, T., Tanaka, K., Kollar, R., Chen, G.C., Ford, R.A., Chan, C.S.M., Takai, Y., and Cabib, E. (1996). Rho1p, a yeast protein at the interface between cell polarization and morphogenesis. Science 272, 277-279.

Erdman, S., Lin, L., Malczynski, M., and Snyder, M. (1998). Pheromoneregulated Genes Required for Yeast Mating Differentiation. J. Cell Biol. 140, 461-483.

Etienne-Manneville, S., and Hall, A. (2002). Rho GTPases in cell biology. Nature $420,629-635$

Fujiwara, T., Tanaka, K., Mino, A., Kikyo, M., Takahashi, K., Shimizu, K., and Takai, Y. (1998). Rho1p-Bni1p-Spa2p interactions: implication in localization of Bnilp at the bud site and regulation of the actin cytoskeleton in Saccharomyces cerevisiae. Mol. Biol. Cell 9, 1221-1233.

Galbraith, C.G., Yamada, K.M., and Sheetz, M.P. (2002). The relationship between force and focal complex development. J. Cell Biol. 159, 695-705.
Gentry, M.S., and Hallberg, R.L. (2002). Localization of Saccharomyces cerevisiae protein phosphatase 2A subunits throughout mitotic cell cycle. Mol. Biol. Cell 13, 3477-3492.

Goyal, R.K., Lin, P., Kanugo, J., Payne, A.S., Muslin, A.J., and Longmore, G.D. (1999). Ajuba, a novel LIM protein, interacts with Grb2, augments mitogenactivated protein kinase activity in fibroblasts, and promotes meiotic maturation of Xenopus oocytes in a Grb2- and Ras-dependent manner. Mol. Cell. Biol. 19, 4379-4389.

Guo, W., Tamanoi, F., and Novick, P. (2001). Spatial regulation of the exocyst complex by Rho1 GTPase. Nat. Cell. Biol. 3, 353-360.

Hall, A. (1998). Rho GTPases and the actin cytoskeleton. Science 279, 509-514.

Hartwell, L.H. (1971). Genetic control of the cell division cycle in yeast. IV. Genes controlling bud emergence and cytokinesis. Exp. Cell. Res. 69, 265-276.

Herskowitz, I., Park, H.-O., Sanders, S., Valtz, N., and Peter, M. (1995). Programming of cell polarity in budding yeast by endogenous and exogenous signals. Cold Spring Harbor Symp. Quant. Biol. 60, 717-727.

Higgins, D.G., and Sharp, P.M. (1988). CLUSTAL: a package for performing multiple sequence alignment on a microcomputer. Gene 73, 237-244.

Iida, H., Nakamura, H., Ono, T., Okumura, M.S., and Anraku, Y. (1994). MID1, a novel Saccharomyces cerevisiae gene encoding a plasma membrane protein, is required for $\mathrm{Ca}_{2}{ }^{+}$influx and mating. Mol. Cell. Biol. 14, 8259-8271.

Jin, H., and Amberg, D.C. (2000). The secretory pathway mediates localization of the cell polarity regulator Aip3p/Bud6p. Mol. Biol. Cell 11, 647-661.

Johnson, D.I. (1999). Cdc 42, an essential rho-type GTPase controlling eukaryotic cell polarity. Microbiol. Mol. Biol. Rev. 63, 54-105.

Jurata, L.W., and Gill, G.N. (1998). Structure and function of LIM domains. Curr. Top. Microbiol. Immunol. 228, 75-113.

Ketela, T., Green, R., and Bussey, H. (1999). Saccharomyces cerevisiae Mid2p is a potential cell wall stress sensor and upstream activator of the PKC1-MPK1 cell integrity pathway. J. Bacteriol. 181, 3330-3340.

Khurana, B., Khurana, T., Khaire, N., and Noegel, A.A. (2002a). Functions of LIM proteins in cell polarity and chemotactic motility. EMBO J. 21, 5331-5342.

Khurana, T., Khurana, B., and Noegel, A.A. (2002b). LIM proteins: association with the actin cytoskeleton. Protoplasma 219, 1-12.

Lorberg, A., Schmitz, H.P., Jacoby, J.J., and Heinisch, J.J. (2001). Lrg1p functions as a putative GTPase-activating protein in the Pkc1p-mediated cell integrity pathway in Saccharomyces cerevisiae. Mol. Genet. Genom. 266, 514526

Madden, K., Sheu, Y.J., Baetz, K., Andrews, B., and Snyder, M. (1997). SBF cell cycle regulator as a target of the yeast PKC-MAP kinase pathway. Science 275, $1781-1784$

Manning, B.D., Padmanabha, R., and Snyder, M. (1997). The Rho-GEF Rom2p localizes to sites of polarized cell growth and participates in cytoskeletal functions in Saccharomyces cerevisiae. Mol. Biol. Cell 8, 1829-1844.

Marquitz, A.R., Harrison, J.C., Bose, I., Zyla, T.R., McMillan, J.N., and Lew, D.J. (2002). The Rho-GAP Bem2p plays a GAP-independent role in the morphogenesis checkpoint. EMBO J. 21, 4012-4025.

Martin, H., Rodriguez-Pachon, J.M., Ruiz, C., Nombela, C., and Molina, M. (2000). Regulatory mechanisms for modulation of signaling through the cell integrity Slt2-mediated pathway in Saccharomyces cerevisiae. J. Biol. Chem. 275, $1511-1519$

Nern, A., and Arkowitz, R.A. (1998). A GTP-exchange factor required for cell orientation. Nature 391, 195-198.

Nern, A., and Arkowitz, R.A. (1999). A Cdc24p-Far1p-gbetagamma protein complex required for yeast orientation during mating. J. Cell Biol. 144, 11871202

Ni, L., and Snyder, M. (2001). A genomic study of the bipolar bud site selection pattern in Saccharomyces cerevisiae. Mol. Biol. Cell 12, 2147-2170.

Nobes, C.D., and Hall, A. (1995). Rho, rac, and cdc42 GTPases regulate the assembly of multimolecular focal complexes associated with actin stress fibers, lamellipodia, and filopodia. Cell 81, 53-62.

Paravicini, G., Cooper, M., Friedli, L., Smith, D.J., Carpentier, J.-L., Klig, L.S., and Payton, M.A. (1992). The osmotic integrity of the yeast cell requires a functional PKC1 gene product. Mol. Cell. Biol. 12, 4896-4905.

Peterson, J., Zheng, Y., Bender, L., Myers, A., Cerione, R., and Bender, A. (1994). Interactions between the bud emergence proteins Bem1p and Bem2p and Rho-type GTPases in yeast. J. Cell Biol. 127, 1395-1406.

Prassler, J., Murr, A., Stocker, S., Faix, J., Murphy, J., and Marriott, G. (1998) DdLIM is a cytoskeleton-associated protein involved in the protrusion of lamellipodia in Dictyostelium. Mol. Biol. Cell 9, 545-559. 
Pringle, J., Bi, E., Harkins, H., Zahner, J., Devirgilio, C., Chant, J., Corado, K., and Fares, H. (1995). Establishment of cell polarity in yeast. Cold Spring Harbor Symp. Quant. Biol. 60, 729-744.

Pruyne, D., and Bretscher, A. (2000). Polarization of cell growth in yeast. I. Establishment and maintenance of polarity states. J. Cell Sci. 113, 365-375.

Qadota, H., Ishii, I., Fujiyama, A., Ohya, Y., and Anraku, Y. (1992). RHO gene products, putative small GTP-binding proteins, are important for activation of the CAL1/CDC43 gene product, a protein geranylgeranyl-transferase in Saccharomyces cerevisiae. Yeast 8, 735-741.

Roumanie, O., Weinachter, C., Larrieu, I., Crouzet, M., and Doignon, F. (2001). Functional characterization of the Bag7, Lrg1 and Rgd2 RhoGAP proteins from Saccharomyces cerevisiae. FEBS Lett. 506, 149-156.

Sadler, I., Crawford, A.W., Michelsen, J.W., and Beckerle, M.C. (1992). Zyxin and CCRP: two interactive LIM domain proteins associated with the cytoskeleton. J. Cell Biol. 119, 1573-1587.

Sambrook, J., Fritsch, E.F., and Maniatis, T. (1989). Molecular Cloning: A Laboratory Manual. Cold Spring Harbor, NY: Cold Spring Harbor Laboratory.

Schmeichel, K.L., and Beckerle, M. (1994). The LIM domain is a modular protein binding interface. Cell 79, 211-219.

Schmidt, A., and Hall, M.N. (1998). Signaling to the actin cytoskeleton. Annu. Rev. Cell Dev. Biol. 14, 305-338.

Schmidt, A., Schmelzle, T., and Hall, M.N. (2002). The RHO1-GAPs SAC7, BEM2 and BAG7 control distinct RHO1 functions in Saccharomyces cerevisiae. Mol. Microbiol. 45, 1433-1441.

Sekiya-Kawasaki, M., Abe, M., Saka, A., Watanabe, D., Kono, K., MinemuraAsakawa, M., Ishihara, S., Watanabe, T., and Ohya, Y. (2002). Dissection of upstream regulatory components of the Rho1p effector, 1,3- $\beta$-glucan synthase, in Saccharomyces cerevisiae. Genetics 162, 663-676.

Sherman, F., Fink, G., and Hicks, J. (1986). Cold Spring Harbor LaboratoriesMethods in Yeast Genetics. Cold Spring Harbor, NY: Cold Spring Harbor Laboratory.

Sikorski, R., and Hieter, P. (1989). A system of shuttle vectors and yeast host strains designed for efficient manipulation of DNA in Saccharomyces cerevisiae. Genetics 122, 19-27.
Smith, G.R., Givan, S.A., Cullen, P., and Sprague, G.F., Jr. (2002). GTPaseactivating proteins for Cdc42. Eukaryotic Cell 1, 469-480.

Stevenson, B.J., Ferguson, B., De Virgilio, C., Bi, E., Pringle, J.R., Ammerer, G., and Sprague, Jr., G.F. (1995). Mutation of RGA1, which encodes a putative GTPase-activating protein for the polarity-establishment protein Cdc42p, activates the pheromone-response pathway in the yeast Saccharomyces cerevisiae. Genes Dev. 9, 2949-2963.

Taira, M., Evrard, J.L., Steinmetz, A., and Dawid, I.B. (1995). Classification of LIM proteins. Trends Genet. 11, 431-432.

Tapon, N., and Hall, A. (1997). Rho, Rac and Cdc42 GTPases regulate the organization of the actin cytoskeleton. Curr. Biol. 9, 86-92.

Toenjes, K.A., Sawyer, M.M., and Johnson, D.I. (1999). The guanine-nucleotide-exchange factor Cdc24p is targeted to the nucleus and polarized growth sites. Curr. Biol. 9, 1183-1186.

Turner, C.E. (2000). Paxillin and focal adhesion signalling. Nat. Cell Biol. 2 E231-E236.

van Drogen, F., and Peter, M. (2002). Spa2p functions as a scaffold-like protein to recruit the Mpk1p MAP kinase module to sites of polarized growth. Curr. Biol. 12, 1698-1703.

Wang, T., and Bretscher, A. (1995). The rho-GAP encoded by BEM2 regulate cytoskeletal structure in budding yeast. Mol. Biol. Cell 6, 1011-1024.

Watanabe, D., Abe, M., and Ohya, Y. (2001). Yeast Lrg1p acts as a specialized RhoGAP regulating 1,3- $\beta$-glucan synthesis. Yeast 18, 943-951.

Winzeler, E.A. et al. (1999). Functional characterization of the S. cerevisiae genome by gene deletion and parallel analysis. Science 285, 901-906.

Zahner, J.E., Harkins, H.A., and Pringle, J.R. (1996). Genetic analysis of the bipolar pattern of bud site selection in the yeast Saccharomyces cerevisiae. Mol. Cell. Biol. 16, 1857-1870.

Zhang, M., Bennett, D., and Erdman, S.E. (2002). Maintenance of mating cell integrity requires the adhesin Fig2p. Eucaryotic Cell 1, 811-822.

Zhao, C., Jung, U.S., Garrett-Engele, P., Roe, T., Cyert, M.S., and Levin, D.E. (1998). Temperature-induced expression of yeast FKS2 is under the dual control of protein kinase C and calcineurin. Mol. Cell. Biol. 18, 1013-1022.

Zheng, Y., Cerione, R., and Bender, A. (1994). Control of the yeast bud-site assembly GTPase Cdc42. Catalysis of guanine nucleotide exchange by Cdc24 and stimulation of GTPase activity by Bem3. J. Biol. Chem. 269, 2369-2372. 